PHYSICAL REVIEW D 80, 084043 (2009)

\title{
Comparison of post-Newtonian templates for compact binary inspiral signals in gravitational-wave detectors
}

\author{
Alessandra Buonanno, ${ }^{1, *}$ Bala R. Iyer, ${ }^{2,3, \dagger}$ Evan Ochsner, ${ }^{1,+}$ Yi Pan, ${ }^{1, \S}$ and B. S. Sathyaprakash ${ }^{3, \|}$ \\ ${ }^{1}$ Maryland Center for Fundamental Physics, Department of Physics, University of Maryland, College Park, Maryland 20742, USA \\ ${ }^{2}$ Raman Research Institute, Bangalore, 560 080, India \\ ${ }^{3}$ School of Physics and Astronomy, Cardiff University, 5, The Parade, Cardiff, United Kingdom, CF24 3YB
}

(Received 6 July 2009; published 29 October 2009)

\begin{abstract}
The two-body dynamics in general relativity has been solved perturbatively using the post-Newtonian (PN) approximation. The evolution of the orbital phase and the emitted gravitational radiation are now known to a rather high order up to $\mathcal{O}\left(v^{8}\right), v$ being the characteristic velocity of the binary. The orbital evolution, however, cannot be specified uniquely due to the inherent freedom in the choice of parameter used in the PN expansion, as well as the method pursued in solving the relevant differential equations. The goal of this paper is to determine the (dis)agreement between different PN waveform families in the context of initial and advanced gravitational-wave detectors. The waveforms employed in our analysis are those that are currently used by Initial LIGO/Virgo, that is, the time-domain PN models TaylorT1, TaylorT2, TaylorT3, the Fourier-domain representation TaylorF2 (or stationary phase approximant), and the effective-one-body model, and two more recent models, TaylorT4 and TaylorEt. For these models we examine their overlaps with one another for a number of different binaries at 2PN, 3PN, and 3.5PN orders to quantify their differences. We then study the overlaps of these families with the prototype effective-onebody family, currently used by Initial LIGO, calibrated to numerical-relativity simulations to help us decide whether there exist preferred families, in terms of detectability and computational cost, that are the most appropriate as search templates. We conclude that as long as the total mass remains less than a certain upper limit $M_{\text {crit }}$, all template families at 3.5PN order (except TaylorT3 and TaylorEt) are equally good for the purpose of detection. The value of $M_{\text {crit }}$ is found to be $\sim 12 M_{\odot}$ for Initial, Enhanced, and Advanced LIGO. From a purely computational point of view, we recommend that 3.5PN TaylorF2 be used below $M_{\text {crit }}$ and that the effective-one-body model calibrated to numerical-relativity simulations be used for total binary mass $M>M_{\text {crit }}$.
\end{abstract}

DOI: 10.1103/PhysRevD.80.084043

PACS numbers: 04.30.Db, 04.25.Nx, 04.80.Nn, 95.55.Ym

\section{INTRODUCTION}

Sensitivity of several interferometric gravitational-wave detectors has either already reached, or is close to, the design goals that were set more than a decade ago [1-7]. Upgrades that are currently underway and planned for the next four to five years will see their sensitivity improve by factors of a few to an order of magnitude [8]. Coalescing binaries consisting of neutron stars and/or black holes are probably the most promising sources for a first direct detection of gravitational waves. At current sensitivities, initial interferometers are capable of detecting binary neutron star inspirals at distances up to $\sim 30 \mathrm{Mpc}$, the range increasing to $\sim 60 \mathrm{Mpc}$ for enhanced detectors (circa 2009-2011) and $\sim 450 \mathrm{Mpc}$ for advanced detectors (circa 2014+). Binary black holes or a mixed system consisting of a neutron star and a black hole can be detected to a far greater distance depending on the total mass and the mass

\footnotetext{
*buonanno@umd.edu

†bri@rri.res.in

*evano@umd.edu

§ypan@umd.edu

" B.Sathyaprakash@astro.cf.ac.uk
}

ratio. For example, a $(10+10) M_{\odot}$ binary can be detected out to distances of $\sim 160 \mathrm{Mpc}$ by initial detectors and $\sim 2200 \mathrm{Mpc}$ by advanced detectors [9].

The range of interferometric detectors for coalescing binaries is computed by assuming that one can pull the signal out of noise by matched filtering. This in turn means that one is able to follow the phasing of gravitational waves typically to within a fraction of a cycle over the duration of the signal in band. The reason for this optimism comes from the fact that one knows the phase evolution of the signal to a high order in post-Newtonian (PN) formalism [10]. Several authors have assessed whether the accuracy with which the formalism provides the waveforms is good enough for the purpose of detection and parameter estimation [11-26]. The problem, as we shall see below, is complicated since the PN approximation does not lead to a unique model of the phase evolution. Moreover, though PN results are good up to mildly relativistic velocities, the standard PN approximants become less and less accurate in the strongly relativistic regime as one approaches the last stable orbit (LSO). Resummation methods [15] and, in particular, the effective-one-body (EOB) [27-29] extensions of the PN approximants are needed for analytical treatments close to and beyond the LSO. 
The success in numerical-relativity simulations of binary black holes [30-34] now provides results for gravitational waveforms that can be compared to standard PN results and other resummed extensions. On the one hand, the analytical PN results for the inspiral phase of the evolution are needed to calibrate and interpret the numerical-relativity waveforms of coalescence and merger. On the other hand, the numerical-relativity results extend the analytical approximations beyond the inspiral phase and provide the important coalescence and merger phases, producing the strongest signals that are crucial for the detection of binary black holes. However, numerical simulations are still computationally expensive and timeconsuming, and presently only a small region of the parameter space can be explored. Even in the foreseeable future, numerical relativity may not be able to handle the tens of thousands of cycles that are expected from highly asymmetric systems (e.g., a neutron star falling into an intermediate-mass black hole of $100 M_{\odot}$ ) or low-mass symmetric systems (e.g., a binary neutron star). Analytical models that smoothly go from the inspiral through coalescense to quasinormal ringing would be needed, and this has led to phenomenological templates [35-37] and EOB waveforms [36,38-46]. In particular, the recent, improved EOB models $[45,46]$, which also incorporate a multiplicative decomposition of the multipolar waveform into several physically motivated factors supplemented by a suitable hybridization (using test particle results) [47], and an improved treatment of nonquasicircular corrections, show evidence of remarkable success in modeling accurately the numerical-relativity waveforms for different mass ratios.

The emphasis of this work is different. Recently, there have been investigations [48] on the ability of various standard families of PN templates to detect a specific signal model, TaylorEt [49-51], and the often-used TaylorF2 to detect a complete numerical-relativity signal including merger and ringdown [36,37]. Reference [48] modeled the signal by the TaylorEt approximant at 3.5PN order and looked at the effectualness and systematic biases in the estimation of mass parameters for TaylorT1, TaylorT4, and TaylorF2 templates in the LIGO and Virgo detectors. It also looked into the possibility of improving the effectualness by using unphysical values of $\nu$ beyond the maximum value of 0.25 . It was found that the overlaps of a TaylorEt signal with the TaylorT1, TaylorT4, and TaylorF2 templates are smaller than 0.97 and involved for equal-mass systems a large bias in the total mass. For unequal-mass systems higher overlaps can be obtained at the cost of a large bias in mass and symmetric mass ratio $\nu$ and can be further improved by unphysical values of $\nu>0.25$. The templates are more unfaithful with increasing total mass. To detect optimally the complete numerical-relativity signal, including merger and ringdown, Ref. [36] suggested the possibility of using the TaylorF2 template bank with a frequency cutoff $f_{c}$ larger than the usual upper cutoff (i.e., the Schwarzschild LSO) and closer to the fundamental quasinormal mode frequency of the final black hole. Moreover, they proposed to further improve this family by allowing either for unphysical values of $\nu$ or for the inclusion of a pseudo 4PN (p4PN) coefficient in the template phase, calibrated to the numerical simulations. Reference [37] extended the results of Ref. [36] to more accurate numerical waveforms, found that 3.5PN templates are nearly always better and rarely significantly worse than the 2PN templates, and proposed simple analytical frequency cutoffs for both Initial and Advanced LIGOfor example, for Initial LIGO they recommended a strategy using p4PN templates for $M \leq 35 M_{\odot}$ and 3.5PN templates with unphysical values of $\nu$ for larger masses. However, we notice that there is no reason for changing the template bank above $35 M_{\odot}$. Reference [37] could have used the p4PN templates over the entire mass region, if they had not employed in their analysis the p4PN coefficient used in Ref. [36], but had calibrated it to the highly accurate waveforms used in their paper. ${ }^{1}$

In this work our primary focus is on binary systems dominated by early inspiral and on a critical study of the variety of approximants that describe this. Towards this end, in this paper we will provide a sufficiently exhaustive comparison of different PN models of adiabatic inspiral for an illustrative variety of different systems and quantify how (dis)similar they are for the purpose of detection. The choice of the PN models used in this paper is motivated by the fact that they are available in the LIGO Algorithms Library (LAL), and some of them have been used in the searches by Initial LIGO. We also compare all these PN models with one fiducial EOB model calibrated to numerical-relativity simulations [40] to delineate the range of mass values where one must definitely go beyond the inspiral-dominated PN models to a more complete description including plunge and coalescence. The choice of this fiducial, preliminary EOB model is only motivated by the fact that it is the EOB model available in LAL and it is currently used for searches by Initial LIGO. It will be improved in the future using the recent results in Refs. $[45,46]$. We will conclude that for total masses below a certain upper limit $M_{\text {crit }}$, all template families at 3.5PN order (except for TaylorT3 and TaylorEt) are equally good for the purpose of detection. $M_{\text {crit }}$ is found to be $\sim 12 M_{\odot}$ for Initial, Enhanced, and Advanced LIGO. Based solely on computational costs, we recommend that 3.5PN TaylorF2 be used below $M_{\text {crit }}$ and that EOB calibrated to numerical-relativity simulations be used for total binary mass $M>M_{\text {crit }}$.

\footnotetext{
${ }^{1}$ We computed that the p4PN coefficient calibrated to the highly accurate waveforms used in Ref. [37] is $\boldsymbol{Y}=3714$, instead of $y=3923$ as found in Ref. [36].
} 
The paper is organized as follows. In Sec. II we summarize the present status of the PN approximation. In Sec. III we recapitulate for completeness the main PN approximants and try to provide a ready reckoner for the equations describing them and the relevant initial and termination conditions. In Sec. IV we discuss the frequency evolution in each of these models. In Sec. V we discuss overlaps and the maximization used in this work. Sections VI and VII present the results of our analysis related to the effectualness, while Sec. VIII summarizes the results related to the faithfulness. In Sec. IX we summarize our main conclusions. Readers who are interested in the main results of the paper and want to avoid technical details could skip Secs. II, III, IV, and V, read the main results of Secs. VI, VII, and VIII, and mainly focus on Sec. IX.

\section{CURRENT STATUS OF POST-NEWTONIAN APPROXIMATION}

Post-Newtonian approximation computes the evolution of the orbital phase $\phi(t)$ of a compact binary as a perturbative expansion in a small parameter, typically taken as $v=(\pi M F)^{1 / 3}$ (characteristic velocity in the binary), or $x=v^{2}$, although other variants exist. Here $M$ is the total mass of the binary and $F$ the gravitational-wave frequency. In the adiabatic approximation, and for the restricted waveform in which case the gravitational-wave phase is twice the orbital phase, the theory allows the phasing to be specified by a pair of differential equations $\dot{\phi}(t)=v^{3} / M$, $\dot{v}=-\mathcal{F}(v) / E^{\prime}(v)$, where $M$ is the total mass of the system, $\mathcal{F}$ its gravitational-wave luminosity, and $E^{\prime}(v)$ is the derivative of the binding energy with respect to $v$. Different PN families arise because one can choose to treat the ratio $\mathcal{F}(v) / E^{\prime}(v)$ differently starting formally from the same PN order inputs [18]. For instance, one can retain the PN expansions of the luminosity $\mathcal{F}(v)$ and $E^{\prime}(v)$ as they appear (the so-called TaylorT1 model), or expand the rational polynomial $\mathcal{F}(v) / E^{\prime}(v)$ in $v$ to consistent $\mathrm{PN}$ order (the TaylorT4 model), recast as a pair of parametric equations $\phi(v)$ and $t(v)$ (the TaylorT2 model), or the phasing could be written as an explicit function of time $\phi(t)$ (the TaylorT3 model). These different representations are made possible because one is dealing with a perturbative series. Therefore, one is at liberty to "resum" or "reexpand" the series in any way one wishes (as long as one keeps terms to the correct order in the perturbation expansions), or even retain the expression as the quotient of two polynomials and treat them numerically. There is also the freedom of writing the series in a different variable, say (suitably adimensional) $E$ (the so-called TaylorEt model).

In addition to these models, there have been efforts to extend the evolution of a binary beyond what is naturally prescribed by the PN formalism. Let us briefly discuss two reasons why the PN evolution cannot be used all the way up to the merger of the two bodies. PN evolution is based on the so-called adiabatic approximation, according to which the fractional change in the orbital frequency $F_{\text {orb }}$ over each orbital period is negligibly small, i.e. $\dot{F}_{\text {orb }} / F_{\text {orb }}^{2} \ll 1$. This assumption is valid during most of the evolution, but begins to fail as the system approaches the LSO where $f_{\text {LSO }}=\left(6^{3 / 2} \pi M\right)^{-1}$. In some cases, the frequency evolution stops being monotonic and $\dot{f}$ changes from being positive to negative well before reaching the LSO_an indication of the breakdown of the approximation.

From the viewpoint of maximizing the detection potential, one is also interested in going beyond the inspiral phase. The merger and ringdown phases of the evolution, when the luminosity is greatest, cannot be modeled by standard PN approximation. The use of resummation techniques more than a decade ago was followed by the construction of the EOB model [27-29], which has analytically provided the plunge, merger, and ringdown phases of the binary evolution. As mentioned before, more recently, these models have been calibrated to numerical-relativity simulations [36,38-46]. We now have a very reliable EOB model that can be used to model the merger dynamics.

An astronomical binary is characterized by a large number of parameters, some of which are intrinsic to the system (e.g., the masses and spins of the component stars and the changing eccentricity of the orbit) and others that are extrinsic (e.g., source location and orientation relative to the detector). In this paper we will worry about only the detection problem. Furthermore, we will assume that a coincident detection strategy will be followed so that we do not have to worry about the angular parameters such as the direction to the source, the wave's polarization, etc. If binaries start their lives when their separation $r$ is far larger compared to their gravitational radius (i.e., $r \gg G M / c^{2}$ ), by the time they enter the sensitivity band of ground-based detectors, any initial eccentricity would have been lost due to gravitational radiation reaction, which tends to circularize $^{2}$ a binary $[59,60]$. Therefore, we shall consider only systems that are on a quasicircular inspiraling orbit. We shall also neglect spins, which means that we have to worry, in reality, about only the two masses of the component bodies.

Our goal is to explore how (dis)similar the different waveform families are. We do this by computing the (normalized) cross correlation between signals and templates, maximized either only over the extrinsic parameters

\footnotetext{
${ }^{2}$ Though this assumption is justified for the prototypical binaries we focus on in this work, there exist credible astrophysical scenarios that lead to inspiral signals from binaries with nonnegligible eccentricity in the sensitive detector bandwidth. A more involved treatment is then called for and available. See e.g. [52-58].
} 
of the templates (faithfulness) or over the intrinsic and extrinsic parameters of the templates (effectualness), the noise power spectral density (PSD) of the detector serving as a weighting factor in the computation of the correlation (see Sec. V). Our conclusions, therefore, will depend on the masses of the compact stars as well as the detector that we hope to observe the signal with.

The overlaps (i.e., the normalized cross correlation maximized over various parameters and weighted by the noise power spectral density) we shall compute are sensitive to the shape of the noise spectral density of a detector and not on how deep that sensitivity is. Now, the upgrade from initial to advanced interferometers will see improvements in sensitivity not only at a given frequency but over a larger band. Therefore, the agreement between different PN models will be sensitive to the noise spectral density that is used in the inner product. Thus, we will compare the PN families using power spectral densities of initial and advanced interferometric detectors.

We end this brief overview with the following observation. As mentioned earlier, following all present gravitational-wave data analysis pipelines, this paper works only in the restricted wave approximation. This approximation assumes the waveform amplitude to be Newtonian and thus includes only the leading second harmonic of the orbital phase. Higher PN order amplitude terms bring in harmonics of the orbital phase other than the dominant one at twice the orbital frequency. Their effects can be significant [61,62], especially close to merger [45], and they need to be carefully included in future work.

\section{THE PN APPROXIMANTS}

For the convenience of the reader, in this section, we recapitulate the basic formulas for the different PN families from Refs. $[18,19]$. While comparing the expressions below to those in Refs. [18,19], recall $\lambda=-1987 / 3080$ $[63,64]$ and $\Theta=-11831 / 9240[65,66]$. In addition to the evolution equations, we shall also provide initial and final conditions. From the perspective of a data analyst, the initial condition is simply a starting frequency $F_{0}$ and phase $\phi_{0}$, which can be translated, with the help of evolution equations, as conditions on the relevant variables. We shall also give explicit expressions for the evolution of the gravitational-wave frequency, namely, $\dot{F} \equiv d F / d t$, or more precisely, the dimensionless quantity $\dot{F} F^{-2}$, in Sec. IV, where they will be used to study the rate at which the binary coalesces in different PN families, which will help us understand the qualitative difference between them. The contents of this section should act as a single point of resource for anyone who is interested in implementing the waveforms for the purpose of data analysis and other applications.
The basic inputs for all families are the PN expressions for the conserved 3PN energy (per unit total mass) $[63,64,67-70] E_{3}(v)$ and 3.5PN energy flux $[65,66,71-$ 73] $\mathcal{F}_{3.5}(v)$,

$$
\begin{aligned}
E_{3}(v)=- & \frac{1}{2} \nu v^{2}\left[1-\left(\frac{3}{4}+\frac{1}{12} \nu\right) v^{2}-\left(\frac{27}{8}-\frac{19}{8} \nu\right.\right. \\
+ & \left.\frac{1}{24} \nu^{2}\right) v^{4}-\left\{\frac{675}{64}-\left(\frac{34445}{576}-\frac{205}{96} \pi^{2}\right) \nu\right. \\
+ & \left.\left.\frac{155}{96} \nu^{2}+\frac{35}{5184} \nu^{3}\right\} v^{6}\right], \\
\mathcal{F}_{3.5}(v)= & \frac{32}{5} \nu^{2} v^{10}\left[1-\left(\frac{1247}{336}+\frac{35}{12} \nu\right) v^{2}+4 \pi v^{3}\right. \\
& -\left(\frac{44711}{9072}-\frac{9271}{504} \nu-\frac{65}{18} \nu^{2}\right) v^{4} \\
& -\left(\frac{8191}{672}+\frac{583}{24} \nu\right) \pi v^{5}+\left\{\frac{6643739519}{69854400}\right. \\
& +\frac{16}{3} \pi^{2}-\frac{1712}{105} \gamma+\left(\frac{41}{48} \pi^{2}-\frac{134543}{7776}\right) \nu \\
& \left.-\frac{94403}{3024} \nu^{2}-\frac{775}{324} \nu^{3}-\frac{856}{105} \log \left(16 v^{2}\right)\right\} v^{6} \\
& \left.-\left(\frac{16285}{504}-\frac{214745}{1728} \nu-\frac{193385}{3024} \nu^{2}\right) \pi v^{7}\right],
\end{aligned}
$$

where $\gamma=0.577216 \ldots$ is the Euler constant. In the adiabatic approximation one assumes that the orbit evolves slowly so that the fractional change in the orbital velocity $\omega$ over an orbital period is negligibly small. That is, $\frac{\Delta \omega}{\omega} \ll$ 1 , or, equivalently, $\frac{\dot{\omega}}{\omega^{2}} \ll 1$. In this approximation, one expects the luminosity in gravitational waves to come from the change in orbital energy averaged over a period. For circular orbits this means one can use the energy balance equation $\mathcal{F}=-d \mathcal{E} / d t$ where $\mathcal{E}=M E$.

In the adiabatic approximation one can write an equation for the evolution of any of the binary parameters. For instance, the evolution of the orbital separation $r(t)$ can be written as $\dot{r}(t)=\dot{\mathcal{E}} /(d \mathcal{E} / d r)=-\mathcal{F} /(d \mathcal{E} / d r)$. Together with Kepler's law, the energy balance equation can be used to obtain the evolution of the orbital phase ${ }^{3}$ :

$$
\begin{aligned}
\frac{d \phi}{d t}-\frac{v^{3}}{M} & =0, \\
\frac{d v}{d t}+\frac{\mathcal{F}(v)}{M E^{\prime}(v)} & =0,
\end{aligned}
$$

or, equivalently,

\footnotetext{
${ }^{3}$ Recall that the gravitational-wave phase is twice the orbital phase for the restricted waveform and leads to differences in factors of 2 between the equations here for the orbital phase and those in [18] for the gravitational-wave phase.
} 


$$
\begin{gathered}
t(v)=t_{\text {ref }}+M \int_{v}^{v_{\text {ref }}} d v \frac{E^{\prime}(v)}{\mathcal{F}(v)}, \\
\phi(v)=\phi_{\text {ref }}+\int_{v}^{v_{\text {ref }}} d v v^{3} \frac{E^{\prime}(v)}{\mathcal{F}(v)},
\end{gathered}
$$

where $t_{\text {ref }}$ and $\phi_{\text {ref }}$ are integration constants and $v_{\text {ref }}$ is an arbitrary reference velocity.

\section{A. TaylorT1}

The TaylorT1 approximant refers to the choice corresponding to leaving the PN expansions of the luminosity $\mathcal{F}(v)$ and $E^{\prime}(v)$ as they appear in Eq. (3.3) as a ratio of polynomials and solving the differential equations numerically,

$$
\begin{gathered}
\frac{d \phi^{(\mathrm{T} 1)}}{d t}-\frac{v^{3}}{M}=0, \\
\frac{d v}{d t}+\frac{\mathcal{F}(v)}{M E^{\prime}(v)}=0 .
\end{gathered}
$$

In the above $v \equiv v^{(\mathrm{T} 1)}$, but for the sake of notational simplicity we write only $v$; from the context the meaning should be clear. In the formulas of this section, and in the sections that follow, the expressions for $\mathcal{F}(v)[E(v)]$ are to be truncated at relative PN orders 2[2], 3[3], and 3.5[3] to obtain 2PN [18,74-76], 3PN, and 3.5PN [19,65,73] template or signal models, respectively.

To see how to set up initial conditions, refer to Eq. (3.4). Let the initial gravitational-wave frequency be $F_{0}$ or, equivalently, let the initial velocity be $v_{0}=\left(\pi M F_{0}\right)^{1 / 3}$. One normally chooses $t=0$ at $v=v_{0}$. This can be achieved by choosing $v_{\text {ref }}=v_{0}$ and $t_{\text {ref }}=0$ in Eq. (3.4). The initial phase $\phi_{\text {ref }}$ is chosen to be either 0 or $\pi / 2$ in order to construct two orthogonal templates (see Sec. VA for details).

\section{B. TaylorT4}

TaylorT4 was proposed in Ref. [23] and investigated in Refs. [33,38,77], thus many years after the other approximants discussed in this paper were proposed (with the exception of TaylorEt, which is even more recent). However, it is a straightforward extension of TaylorT1, and at 3.5PN order, by coincidence, it is in better agreement with numerical simulations of the inspiral phase $[33,36,38,41,43,50,77]$. The approximant is obtained by expanding the ratio of the polynomials $\mathcal{F}(v) / E^{\prime}(v)$ to the consistent PN order. The equation for $v^{(\mathrm{T} 4)}(t) \equiv v(t)$ at 3.5PN order reads

$$
\begin{aligned}
\frac{d v}{d t}= & \frac{32}{5} \frac{\nu}{M} v^{9}\left[1-\left(\frac{743}{336}+\frac{11}{4} \nu\right) v^{2}+4 \pi v^{3}+\left(\frac{34103}{18144}+\frac{13661}{2016} \nu+\frac{59}{18} \nu^{2}\right) v^{4}-\left(\frac{4159}{672}+\frac{189}{8} \nu\right) \pi v^{5}\right. \\
& +\left(\frac{16447322263}{139708800}+\frac{16}{3} \pi^{2}-\frac{1712}{105} \gamma+\left(\frac{451}{48} \pi^{2}-\frac{56198689}{217728}\right) \nu+\frac{541}{896} \nu^{2}-\frac{5605}{2592} \nu^{3}-\frac{856}{105} \log \left(16 v^{2}\right)\right) v^{6} \\
& \left.-\left(\frac{4415}{4032}-\frac{358675}{6048} \nu-\frac{91495}{1512} \nu^{2}\right) \pi v^{7}\right] .
\end{aligned}
$$

The orbital phase $\phi^{(\mathrm{T} 4)}$ is determined, as in the case of TaylorT1, by Eq. (3.3a), and the numerical solution of Eqs. (3.3a) and (3.6) yields the TaylorT4 approximant.

Note that although TaylorT1 and TaylorT4 are perturbatively equivalent, the evolution of the phase can be quite different in these two approximations. The asymptotic structures of the approximants are also quite different: While $\dot{v}$ can have a pole (although not necessarily in the region of interest) when using Eq. (3.5b), no pole is possible when Eq. (3.6) is used. Differences of this kind can, in principle, mean that the various $\mathrm{PN}$ families give different phasing of the orbit. The hope is that when the PN order up to which the approximation is known is large, then the difference between the various PN families becomes negligible.

Setting up the initial conditions for TaylorT4 is the same as in the case of TaylorT1.

\section{TaylorT2}

TaylorT2 is based on the second form of the phasing relations, Eq. (3.4). Expanding the ratio of the polynomials $\mathcal{F}(v) / E^{\prime}(v)$ in these equations to consistent PN order and integrating them, one obtains a pair of parametric equations for $\phi(v)$ and $t(v)$, the TaylorT2 model.

$$
\begin{gathered}
\phi_{n / 2}^{(\mathrm{T} 2)}(v)=\phi_{\mathrm{ref}}^{(\mathrm{T} 2)}+\phi_{N}^{v}(v) \sum_{k=0}^{n} \hat{\phi}_{k}^{v} v^{k}, \\
t_{n / 2}^{(\mathrm{T} 2)}(v)=t_{\mathrm{ref}}^{(\mathrm{T} 2)}+t_{N}^{v}(v) \sum_{k=0}^{n} \hat{t}_{k}^{v} v^{k} .
\end{gathered}
$$

Of all the models considered in this study, TaylorT2 is computationally the most expensive. This is because the phase evolution involves solving a pair of transcendental equations, which is very time-consuming. 


$$
\begin{aligned}
\phi_{3.5}^{(\mathrm{T} 2)}(v)= & \phi_{\mathrm{ref}}^{(\mathrm{T} 2)}-\frac{1}{32 \nu v^{5}}\left[1+\left(\frac{3715}{1008}+\frac{55}{12} \nu\right) v^{2}-10 \pi v^{3}+\left(\frac{15293365}{1016064}+\frac{27145}{1008} \nu+\frac{3085}{144} \nu^{2}\right) v^{4}+\left(\frac{38645}{672}-\frac{65}{8} \nu\right)\right. \\
& \times \ln \left(\frac{v}{v_{\mathrm{lso}}}\right) \pi v^{5}+\left\{\frac{12348611926451}{18776862720}-\frac{160}{3} \pi^{2}-\frac{1712}{21} \gamma+\left(\frac{2255}{48} \pi^{2}-\frac{15737765635}{12192768}\right) \nu+\frac{76055}{6912} \nu^{2}\right. \\
& \left.\left.-\frac{127825}{5184} \nu^{3}-\frac{856}{21} \log \left(16 v^{2}\right)\right\} v^{6}+\left(\frac{77096675}{2032128}+\frac{378515}{12096} \nu-\frac{74045}{6048} \nu^{2}\right) \pi v^{7}\right], \\
t_{3.5}^{(\mathrm{T} 2)}(v)= & t_{\mathrm{ref}}^{(\mathrm{T} 2)}-\frac{5 M}{256 \nu v^{8}}\left[1+\left(\frac{743}{252}+\frac{11}{3} \nu\right) v^{2}-\frac{32}{5} \pi v^{3}+\left(\frac{3058673}{508032}+\frac{5429}{504} \nu+\frac{617}{72} \nu^{2}\right) v^{4}-\left(\frac{7729}{252}-\frac{13}{3} \nu\right) \pi v^{5}\right. \\
& +\left\{-\frac{10052469856691}{23471078400}+\frac{128}{3} \pi^{2}+\frac{6848}{105} \gamma+\left(\frac{3147553127}{3048192}-\frac{451}{12} \pi^{2}\right) \nu-\frac{15211}{1728} \nu^{2}+\frac{25565}{1296} \nu^{3}\right. \\
& \left.\left.+\frac{3424}{105} \log \left(16 v^{2}\right)\right\} v^{6}+\left(-\frac{15419335}{127008}-\frac{75703}{756} \nu+\frac{14809}{378} \nu^{2}\right) \pi v^{7}\right] .
\end{aligned}
$$

In this case, $t_{\text {ref }}$ has to be chosen so that $t=0$ when $F=$ $F_{0}$ or $v=v_{0}$. This can be achieved most simply by solving for $t_{\text {ref }}$, using Eq. (3.8b), substituting $v=v_{0}$ on the righthand side and putting the left side to zero.

\section{TaylorT3}

This form of the approximant goes a step further than the previous TaylorT2 approximant. After computing as before a parametric representation of the phasing formula $\phi(v)$ and $t(v)$, one explicitly inverts $t(v)$ to obtain $v(t)$ and uses it to produce an explicit representation of $\phi(t) \equiv \phi(v(t)))$.
This is the TaylorT3 approximant:

$$
\begin{aligned}
& \phi_{n / 2}^{(\mathrm{T} 3)}(t)=\phi_{\mathrm{ref}}^{(\mathrm{T} 3)}+\phi_{N}^{t} \sum_{k=0}^{n} \hat{\phi}_{k}^{t} \theta^{k}, \\
& F_{n / 2}^{(\mathrm{T} 3)}(t)=F_{N}^{t} \sum_{k=0}^{n} \hat{F}_{k}^{t} \theta^{k},
\end{aligned}
$$

where $\theta=\left[\nu\left(t_{\text {ref }}-t\right) /(5 M)\right]^{-1 / 8}$ and $F \equiv(2 d \phi / d t) \times$ $(2 \pi)^{-1}=v^{3} /(\pi M)$ is the instantaneous gravitationalwave frequency.

$$
\begin{aligned}
\phi_{3.5}^{(\mathrm{T} 3)}(t)= & \phi_{\mathrm{ref}}^{(\mathrm{T} 3)}-\frac{1}{\nu \theta^{5}}\left[1+\left(\frac{3715}{8064}+\frac{55}{96} \nu\right) \theta^{2}-\frac{3 \pi}{4} \theta^{3}+\left(\frac{9275495}{14450688}+\frac{284875}{258048} \nu+\frac{1855}{2048} \nu^{2}\right) \theta^{4}+\left(\frac{38645}{21504}-\frac{65}{256} \nu\right)\right. \\
& \times \ln \left(\frac{\theta}{\theta_{\mathrm{lso}}}\right) \pi \theta^{5}+\left\{\frac{831032450749357}{57682522275840}-\frac{53}{40} \pi^{2}+\left(-\frac{126510089885}{4161798144}+\frac{2255}{2048} \pi^{2}\right) \nu-\frac{107}{56} \gamma+\frac{154565}{1835008} \nu^{2}\right. \\
& \left.\left.-\frac{1179625}{1769472} \nu^{3}-\frac{107}{56} \log (2 \theta)\right\} \theta^{6}+\left(\frac{188516689}{173408256}+\frac{488825}{516096} \nu-\frac{141769}{516096} \nu^{2}\right) \pi \theta^{7}\right], \\
F_{3.5}^{(\mathrm{T} 3)}(t)= & \frac{\theta^{3}}{8 \pi M}\left[1+\left(\frac{743}{2688}+\frac{11}{32} \nu\right) \theta^{2}-\frac{3}{10} \pi \theta^{3}+\left(\frac{1855099}{14450688}+\frac{56975}{258048} \nu+\frac{371}{2048} \nu^{2}\right) \theta^{4}-\left(\frac{7729}{21504}-\frac{13}{256} \nu\right) \pi \theta^{5}\right. \\
& +\left\{-\frac{720817631400877}{288412611379200}+\frac{53}{200} \pi^{2}+\frac{107}{280} \gamma+\left(\frac{25302017977}{4161798144}-\frac{451}{2048} \pi^{2}\right) \nu-\frac{30913}{1835008} \nu^{2}+\frac{235925}{1769472} \nu^{3}\right. \\
& \left.\left.+\frac{107}{280} \log (2 \theta)\right\} \theta^{6}+\left(-\frac{188516689}{433520640}-\frac{97765}{258048} \nu+\frac{141769}{1290240} \nu^{2}\right) \pi \theta^{7}\right] .
\end{aligned}
$$

The initial conditions in this case are slightly more complicated than in the previous cases. Given an initial frequency $F_{0}$, one numerically solves Eq. (3.10b) to find the value of $t_{\text {ref }}$ at which $F=F_{0}$ and $t=0$ (recall that $\theta$ involves $t_{\text {ref }}$.) Note that as $t \rightarrow t_{\text {ref }}$, formally $F \rightarrow$ diverges.

\section{E. TaylorEt}

The TaylorEt was recently introduced in Refs. [49-51]. Introducing ${ }^{4} \zeta=-2 E / \nu$ (recall that our $E$ is conserved

\footnotetext{
${ }^{4}$ Note that the $\zeta$ in this paper is denoted variously by $\zeta$ in [49] but by $\xi$ in e.g. [48].
}

energy per total mass), the TaylorEt approximants are obtained starting from Eq. (3.1) for $E(x)$ or $\zeta(x)$ and inverting it to obtain $x(\zeta)$ :

$$
\begin{aligned}
x= & \zeta\left[1+\left(\frac{3}{4}+\frac{1}{12} \nu\right) \zeta+\left(\frac{9}{2}-\frac{17}{8} \nu+\frac{1}{18} \nu^{2}\right) \zeta^{2}\right. \\
& \left.+\left(\frac{405}{16}+\left(\frac{205}{96} \pi^{2}-\frac{4795}{72}\right) \nu+\frac{55}{64} \nu^{2}+\frac{35}{1296} \nu^{3}\right) \zeta^{3}\right] .
\end{aligned}
$$

With this choice of variable the equation determining the evolution of $v$, Eq. (3.3b), transforms to the balance equation for $E$ rewritten in terms of the $\zeta$ variable: 


$$
\frac{d \zeta}{d t}=\frac{2 \mathcal{F}(v(\zeta))}{\nu M} .
$$

There is no difference between $\mathrm{T} 1$ and $\mathrm{T} 4$ approximants in the Et parametrization, and the gravitational-wave phasing equations (3.3a) and (3.3b) in terms of $\zeta$ become [48]

$$
\begin{aligned}
\begin{aligned}
d \phi^{(\mathrm{Et})}(t) \\
d t
\end{aligned}= & \frac{\zeta^{3 / 2}}{M}\left[1+\left(\frac{9}{8}+\frac{1}{8} \nu\right) \zeta+\left(\frac{891}{128}-\frac{201}{64} \nu+\frac{11}{128} \nu^{2}\right) \zeta^{2}\right. \\
& \left.+\left\{\frac{41445}{1024}-\left(\frac{309715}{3072}-\frac{205}{64} \pi^{2}\right) \nu+\frac{1215}{1024} \nu^{2}+\frac{45}{1024} \nu^{3}\right\} \zeta^{3}\right], \\
\frac{d \zeta}{d t}= & \frac{64 \nu \zeta^{5}}{5 M}\left[1+\left(\frac{13}{336}-\frac{5}{2} \nu\right) \zeta+4 \pi \zeta^{3 / 2}+\left(\frac{117857}{18144}-\frac{12017}{2016} \nu+\frac{5}{2} \nu^{2}\right) \zeta^{2}+\left(\frac{4913}{672}-\frac{177}{8} \nu\right) \pi \zeta^{5 / 2}\right. \\
& +\left(\frac{37999588601}{279417600}+\frac{16}{3} \pi^{2}-\frac{1712}{105} \gamma+\left(\frac{369}{32} \pi^{2}-\frac{24861497}{72576}\right) \nu+\frac{488849}{16128} \nu^{2}-\frac{85}{64} \nu^{3}-\frac{856}{105} \log (16 \zeta)\right) \zeta^{3}
\end{aligned}
$$

\section{F. TaylorF2}

The most commonly used form of the approximant is the Fourier representation computed using the stationary phase approximation (SPA). Using the SPA the waveform in the frequency domain may be written as

$$
\begin{aligned}
\tilde{h}^{\mathrm{spa}}(f) & =\frac{a\left(t_{f}\right)}{\sqrt{\dot{F}\left(t_{f}\right)}} e^{i\left[\psi_{f}\left(t_{f}\right)-\pi / 4\right]}, \\
\psi_{f}(t) & \equiv 2 \pi f t-2 \phi(t),
\end{aligned}
$$

where $t_{f}$ is the saddle point defined by solving for $t$, $d \psi_{f}(t) / d t=0$, i.e. the time $t_{f}$ when the gravitationalwave frequency $F(t)$ becomes equal to the Fourier variable $f$. In the adiabatic approximation [denoting $v_{f} \equiv$ $(\pi M f)^{1 / 3}$ ] the values of $t_{f}$ and $\psi_{f}\left(t_{f}\right)$ are given by the following integrals:
As in the time-domain case, it is more efficient to use the equivalent differential form

$$
\frac{d \psi}{d f}-2 \pi t=0, \quad \frac{d t}{d f}+\frac{\pi M^{2}}{3 v^{2}} \frac{E^{\prime}(f)}{\mathcal{F}(f)}=0,
$$

and this characterizes the TaylorF1 approximant.

The analogue of the TaylorT2 in the frequency domain follows by explicitly truncating the energy and flux functions to consistent post-Newtonian orders and explicating the $v$ integration in the above. This leads us to a Fourier domain waveform, the TaylorF2, which is the most often employed PN approximant, given by

$$
\tilde{h}(f)=\mathcal{A} f^{-7 / 6} e^{i \psi(f),}
$$

where $\mathcal{A} \propto \mathcal{M}^{5 / 6} Q$ (angles) $/ D$, and $D$ is the distance to the binary. To 3.5PN order the phase of the Fourier domain waveform is given by

$$
\begin{aligned}
\psi_{3.5}^{(\mathrm{F} 2)}(f)= & 2 \pi f t_{c}-\phi_{c}-\frac{\pi}{4}+\frac{3}{128 \nu v^{5}}\left[1+\frac{20}{9}\left(\frac{743}{336}+\frac{11}{4} \nu\right) v^{2}-16 \pi v^{3}+10\left(\frac{3058673}{1016064}+\frac{5429}{1008} \nu+\frac{617}{144} \nu^{2}\right) v^{4}\right. \\
& +\pi\left(\frac{38645}{756}-\frac{65}{9} \nu\right)\left\{1+3 \log \left(\frac{v}{v_{\mathrm{lso}}}\right)\right\} v^{5}+\left\{\frac{11583231236531}{4694215680}-\frac{640}{3} \pi^{2}-\frac{6848 \gamma}{21}-\frac{6848}{21} \log (4 v)\right. \\
& \left.+\left(-\frac{15737765635}{3048192}+\frac{2255 \pi^{2}}{12}\right) \nu+\frac{76055}{1728} \nu^{2}-\frac{127825}{1296} \nu^{3}\right\} v^{6} \\
& \left.+\pi\left(\frac{77096675}{254016}+\frac{378515}{1512} \nu-\frac{74045}{756} \nu^{2}\right) v^{7}\right]
\end{aligned}
$$

where $v=(\pi M f)^{1 / 3}$.

In this case one has to specify the constants $t_{c}$ and $\phi_{c}$, and they can be chosen arbitrarily. 


\section{G. The effective-one-body model}

In this paper since we are not particularly concerned with the coalescence signal, we employ the less sophisticated earlier version of the EOB model calibrated to numerical-relativity simulations from Ref. [40] (for more sophisticated versions of the EOB model see Refs. [4246]). Below we briefly review the EOB model from Ref. [40].

Introducing polar coordinates $(r, \phi)$ and their conjugate momenta $\left(p_{r}, p_{\phi}\right)$, the EOB effective metric takes the form [27]

$$
d s_{\mathrm{eff}}^{2}=-A(r) d t^{2}+\frac{D(r)}{A(r)} d r^{2}+r^{2}\left(d \theta^{2}+\sin ^{2} \theta d \phi^{2}\right) .
$$

The EOB Hamiltonian reads

$$
H^{\text {real }}\left(r, p_{r}, p_{\phi}\right) \equiv \mu \hat{H}^{\text {real }}=M \sqrt{1+2 \nu\left(\frac{H^{\mathrm{eff}}-\mu}{\mu}\right)},
$$

with the effective Hamiltonian [27,29]

$$
\begin{aligned}
H^{\mathrm{eff}}\left(r, p_{r}, p_{\phi}\right) & \equiv \mu \hat{H}^{\mathrm{eff}} \\
& =\mu \sqrt{A(r)\left[1+\frac{A(r)}{D(r)} p_{r}^{2}+\frac{p_{\phi}^{2}}{r^{2}}+2(4-3 \nu) \nu \frac{p_{r}^{4}}{r^{2}}\right] .}
\end{aligned}
$$

The Taylor approximants to the coefficients $A(r)$ and $D(r)$ can be written as $[27,29]$

$$
\begin{aligned}
& A_{k}(r)=\sum_{i=0}^{k+1} \frac{a_{i}(\nu)}{r^{i}}, \\
& D_{k}(r)=\sum_{i=0}^{k} \frac{d_{i}(\nu)}{r^{i}} .
\end{aligned}
$$

The functions $A(r), D(r), A_{k}(r)$, and $D_{k}(r)$ all depend on the symmetric mass ratio $\nu$ through the $\nu$-dependent coefficients $a_{i}(\nu)$ and $d_{i}(\nu)$. These coefficients are currently known through 3PN order (i.e. up to $k=4$ ) and can be read from Ref. [40]. During the last stages of inspiral and plunge, ${ }^{5}$ the EOB dynamics can be adjusted closer to the numerical simulations by including in the radial potential $A(r)$ a p4PN coefficient $a_{5}(\nu)$, and $a_{5}(\nu)=\lambda_{0} \nu$, with $\lambda_{0}$ a constant. ${ }^{6}$ In order to assure the presence of a horizon in the

\footnotetext{
${ }^{5}$ To deal with the steep rise of various quantities during the plunge, it is advantageous to consider the EOB equations in terms of the tortoise radial coordinate $r_{*}$ and its conjugate $p_{r_{*}}$ rather than in terms of the standard radial coordinate $r$ and $p_{r}$ as above. The form of $H^{\text {eff }}$ in the two cases will be different [39]. For the level of accuracy in our present work, this difference is irrelevant.

${ }^{6}$ Note that $\lambda_{0}$ was denoted $\lambda$ in Ref. [40], and $a_{5}$ in Refs. [39,41,43,44].
}

effective metric (3.19), a zero needs to be factored out from $A(r)$. This is obtained by applying a Padé resummation [29]. The Padé coefficients for the expansion of $A(r)$ and $D(r)$ at $\mathrm{p} 4 \mathrm{PN}$ order are denoted $A_{4}^{1}(r)$ and $D_{4}^{0}(r)$, and their explicit form can be read from Ref. [40].

The EOB Hamilton equations are written in terms of the reduced (i.e., dimensionless) quantities $\hat{H}^{\text {real }}$ [defined in Eq. (3.20)], $\hat{t}=t / M$, and $\hat{\omega}=\omega M[28]$ :

$$
\begin{aligned}
\frac{d r}{d \hat{t}} & =\frac{\partial \hat{H}^{\text {real }}}{\partial p_{r}}\left(r, p_{r}, p_{\phi}\right), \\
\frac{d \phi}{d \hat{t}} & =\frac{\partial \hat{H}^{\text {real }}}{\partial p_{\phi}}\left(r, p_{r}, p_{\phi}\right), \\
\frac{d p_{r}}{d \hat{t}} & =-\frac{\partial \hat{H}^{\text {real }}}{\partial r}\left(r, p_{r}, p_{\phi}\right), \\
\frac{d p_{\phi}}{d \hat{t}} & =\hat{\mathcal{F}}_{\phi}\left(r, p_{r}, p_{\phi}\right),
\end{aligned}
$$

with the definition $\hat{\omega} \equiv d \phi / d \hat{t}$. Another critical input to the EOB model is the form for the radiation-reaction force arising from the basic PN expression of the energy flux. Different choices include Padé resummation [15], and the more recent $\rho_{\ell m}$ resummation [47]. It also further includes the introduction of terms describing next-to-quasicircular effects. Here, for the $\phi$ component of the radiationreaction force, we use the less sophisticated Keplerian Padé approximant to the energy flux as given by Eq. (15) of Ref. [40].

The inspiral-plunge EOB waveform at leading order in a PN expansion reads

$$
h^{\text {insp-plunge }}(t) \equiv \hat{\omega}^{1 / 3} \cos [2 \phi(t)] .
$$

The merger-ringdown waveform in the EOB approach is built as a superposition of quasinormal modes [28,3841,78], as

$$
h^{\text {merger-RD }}(t)=\sum_{n=0}^{N-1} A_{n} e^{-i \sigma_{n}\left(t-t_{\text {match }}\right)},
$$

where $n$ is the overtone number of the Kerr quasinormal mode, $N$ is the number of overtones included in our model, and $A_{n}$ are complex amplitudes to be determined by a matching procedure described below. The quantities $\sigma_{n}=$ $\omega_{n}-i \alpha_{n}$, where the oscillation frequencies $\omega_{n}>0$ and the inverse decay times $\alpha_{n}>0$, are numbers associated with each quasinormal mode. The complex frequencies are known functions of the final black-hole mass and spin and can be found in Ref. [79]. The final black-hole masses and spins are obtained from the fitting to numerical results worked out in Ref. [40].

The complex amplitudes $A_{n}$ in Eq. (3.25) are determined by matching the EOB merger-ringdown waveform with the EOB inspiral-plunge waveform close to the EOB light ring. In particular, in Ref. [40] the matching point is provided analytically by Eq. (37). In order to do this, $N$ independent 
complex equations are needed. The $N$ equations are obtained at the matching time by imposing continuity of the waveform and its time derivatives,

$$
\begin{aligned}
\frac{d^{k}}{d t^{k}} h^{\text {insp-plunge }}\left(t_{\text {match }}\right) & =\frac{d^{k}}{d t^{k}} h^{\text {merger-RD }}\left(t_{\text {match }}\right) \\
(k & =0,1,2, \cdots, N-1) .
\end{aligned}
$$

In this paper we use $N=3$. The above matching approach is referred to as point matching. It gives better smoothness around the matching time, but it is not very stable numerically when $N$ is large and higher-order numerical derivatives are needed. More sophisticated matching procedures have been proposed in the literature to overcome the stability issue. Reference [39] introduced the comb matching approach, where $N$ equations are obtained at $N$ points evenly sampled in a small time interval $\Delta t_{\text {match }}$ centered at $t_{\text {match }}$. More recently, to improve the smoothness of the comb matching, Ref. [46] introduced the hybrid comb matching approach, where one chooses a time interval $\Delta t_{\text {match }}$ ending at $t_{\text {match }}$, and imposes not only the continuity of the waveform at $N-4$ points evenly sampled from $t_{\text {match }}-\Delta t_{\text {match }}$ to $t_{\text {match }}$, but also the continuity of the first and second order time derivatives of the waveform at $t_{\text {match }}-\Delta t_{\text {match }}$ and $t_{\text {match }}$.

Finally, the full (inspiral-plunge-merger-ringdown) EOB waveform reads

$$
h(t)=h^{\text {insp-plunge }}(t) \theta\left(t_{\text {match }}-t\right)+h^{\text {merger-RD }} \theta\left(t-t_{\text {match }}\right),
$$

where we denote with $\theta$ the Heaviside step function.

\section{H. Waveforms and termination conditions}

Before concluding this section we note a few other points concerning the generation of the waveform. Since our goal is to study the agreement between different waveforms, it is not necessary to separately consider the two different polarizations, but only the detector response. For time-domain models TaylorT1, TaylorT2, TaylorT3, TaylorT4, and EOB, the waveform is taken as

$$
h_{\mathrm{A}}(t)=C v_{\mathrm{A}}^{2} \sin \left[2 \phi_{\mathrm{A}}(t)\right],
$$

where $v_{\mathrm{A}}$ and $\phi_{\mathrm{A}}(t)$ are computed using the relevant formulas corresponding to the approximant $\mathrm{A}$. In the case of TaylorEt the waveform is taken to be

$$
h_{\mathrm{Et}}(t)=C \zeta(t) \sin \left[2 \phi_{\mathrm{Et}}(t)\right] .
$$

In all cases the constant $C$ is fixed by demanding that the norm of the signal be unity (cf. Sec. V). The initial phase of the signal is set to 0, while in the case of templates we construct two orthonormal waveforms corresponding to the starting phases of 0 and $\pi / 2$.

The waveforms are terminated when $v$ reaches the value quoted in Table I or before, if the frequency evolution is not monotonic (see next section). For instance, in the case of
TABLE I. The termination condition for waveform generation is chosen to be either LSO corresponding to the Schwarzschild metric $v_{S}=6^{-1 / 2}$, or the extremum defined by the $\mathrm{P}$ approximant of the energy function as in [15], which is $v_{P_{4}}$ at $2 \mathrm{PN}$ and $v_{P_{6}}$ at $3 \mathrm{PN}$ and $3.5 \mathrm{PN}$. In the case of TaylorT3 at 3.5PN, as the frequency evolution is not monotonic, the evolution has to be terminated prematurely at $v_{m}$ such that $\dot{F}\left(v_{m}\right)=0$.

\begin{tabular}{lcccccc}
\hline \hline Order/Approx. & $\mathrm{T} 1$ & $\mathrm{~T} 2$ & $\mathrm{~T} 3$ & $\mathrm{~T} 4$ & $\mathrm{Et}$ & $\mathrm{F} 2$ \\
\hline 2PN & $v_{S}$ & $v_{S}$ & $v_{S}$ & $v_{P_{4}}$ & $v_{P_{4}}$ & $v_{P_{4}}$ \\
3PN & $v_{S}$ & $v_{S}$ & $v_{S}$ & $v_{P_{6}}$ & $v_{P_{6}}$ & $v_{P_{6}}$ \\
3.5PN & $v_{S}$ & $v_{S}$ & $v_{m}$ & $v_{P_{6}}$ & $v_{P_{6}}$ & $v_{P_{6}}$ \\
\hline \hline
\end{tabular}

TaylorT3 at 3.5PN order the approximant has an unusual behavior whereby the frequency evolution ceases to be monotonic well before $v$ reaches the nominal value of $1 / \sqrt{6}$. In the case of TaylorT1, TaylorT2, and TaylorT3, the termination is at the LSO defined by the Schwarzschild metric, namely, $v=1 / \sqrt{6}$, at all PN orders, but we also check for monotonicity of the frequency evolution. For other approximants, except EOB, we terminate at the extremum of the P-approximant energy function [15]. In the case of EOB, the waveform is terminated at the end of the quasinormal ringing.

\section{FREQUENCY EVOLUTION}

The quantity that determines the evolution of a binary, its phasing, and the duration for which it lasts, starting from a particular frequency, is the acceleration of the bodies under radiation reaction. Equivalently, it is the evolution of the derivative of the gravitational-wave frequency $\dot{F}=d F / d t$ which determines the phasing of the waves. When the separation between the bodies is large, the frequency evolution is slow and the quantity [18] $\epsilon(t)=\dot{F} F^{-2}$, which measures the fractional change in the frequency over a period, is small: $\dot{F} F^{-2} \ll 1$. As the binary evolves, this quantity increases but, as seen in numerical evolutions, remains finite and positive all the way up to the merger of the two bodies. In what follows we will explore the behavior of $\epsilon$ as a function of the PN parameter $v$ rather than $t$, because the former parameter is (mass) scale-free, unlike the latter.

Computing the adiabaticity parameter $\epsilon(v)$ in the case of TaylorT1 and TaylorT4 is straightforward using Eqs. (3.5b) and (3.6). In the case of TaylorT2, one differentiates Eq. (3.8b) with respect to $v$ and then takes its reciprocal. Finding $\epsilon(v)$ in the case of TaylorEt is more involved. The frequency $F$ is given by Eq. (3.13a) but the righthand side is a function of $\zeta$. One must, therefore, combine Eqs. (3.13a) and (3.13b) to find the derivative of the frequency:

$$
\pi \dot{F}=\pi \frac{d F}{d \zeta} \frac{d \zeta}{d t}=\frac{d}{d \zeta}\left(\frac{d \phi}{d t}\right) \frac{d \zeta}{d t}
$$



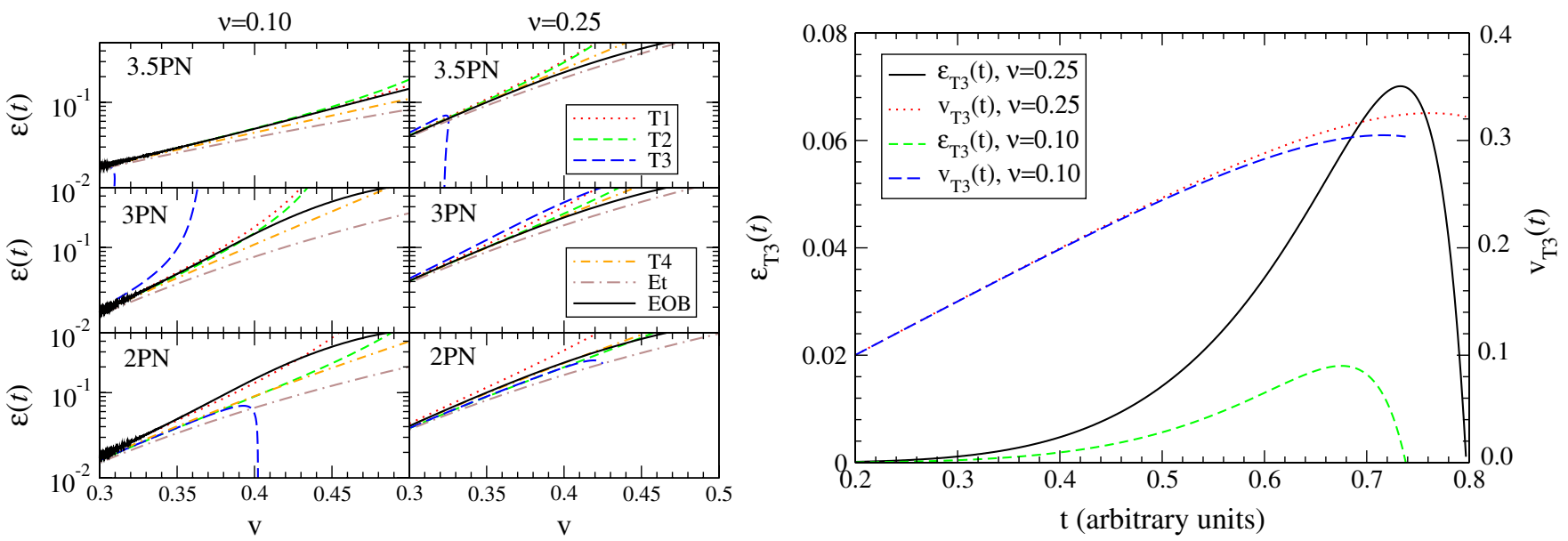

FIG. 1 (color online). On the left panel the plots show the evolution of frequency in different PN families. The adiabaticity parameter $\epsilon(t) \equiv F^{-2} \dot{F}$ is essentially the same for all the different approximations at $v \ll 1$. As the binary gets close to coalescence the various approximations begin to differ from each other. The right panel shows the adiabaticity parameter for the TaylorT3 model as a function of time $t$ at 3.5PN order. Note that $\epsilon_{\mathrm{T} 3}(t)$ begins to decrease and even becomes less than zero before $v$ reaches its nominal value of $1 / \sqrt{6}$. This leads to waveforms that are significantly shorter in the case of TaylorT3.

The above equation still gives $\dot{F}$ as a function of $\zeta$. One can then use Eq. (3.11) to get $\epsilon(v)$. Consequently, there is no guarantee that $v$ will be monotonic in the region of interest. However, we do find that the function $\epsilon_{\mathrm{Et}}(v)$ is positive in the region of interest, and therefore $v$ increases monotonically for TaylorEt. To find $\epsilon(v)$ for TaylorT3, $\dot{F}$ is given by differentiating Eq. (3.10b) with respect to $t$ [recall $\theta=$ $\theta(t)]$, and then one uses the same equation to find $v=$ $(\pi M F)^{1 / 3}$ at a given $t$. It turns out that for TaylorT3 the function $\epsilon_{\mathrm{T} 3}$ can become negative in the region of interest (exactly when this happens depends on the PN order and mass ratio) and so $v$ does not generally increase monotonically.

Figure 1, left panel, plots $\epsilon(v)$ for two values of the mass ratio: $\nu=0.10$ and $\nu=0.25$. When $v$ is small $(v \ll$ $1 / \sqrt{6}) \epsilon(v)$ for the different approximants is the same. Therefore, in systems for which $v$ remains small when the signal is in band (as, for example, in a binary neutron star), the different approximants, as we shall see in the next section, agree well with each other. As $v$ approaches $1 / \sqrt{6}$, different approximations tend to differ greatly, which means we cannot expect good agreement between the different PN families. Of the approximants considered here, TaylorEt seems to have the smallest value of $\epsilon(v)$ at any given $v$. Therefore, the evolution will be slower, and the duration of the waveform from a given frequency larger, than the other approximants [51]. TaylorT3 also differs from all others because $\epsilon(v)$ becomes negative before the last stable orbit, and so $v$ does not generally increase monotonically for this approximant. This behavior can be seen at 2PN and 3.5PN orders in the left panel of Fig. 1. The reason for this can be seen in Fig. 1, right panel, where we have shown the time development of $\epsilon_{\mathrm{T} 3}(t)$ for two values of $\nu=0.10,0.25$. Since $\dot{F}$ becomes negative before reaching the last stable orbit, the waveform has to be terminated before $v$ reaches $1 / \sqrt{6}$.

\section{EFFECTUALNESS}

The goal of this study is to compare the different PN approximations by measuring their mutual effectualness (i.e., overlaps maximized over intrinsic and extrinsic parameters) for a number of different mass pairs. To this end it will be very useful to define the scalar product of waveforms. Given waveforms $h_{k}$ and $q_{k}, k=0, \ldots, N-1$, where $h_{k}$ is the $k$ th sample of the signal $h(t)$ at time $t_{k}=$ $k \Delta, \Delta=1 / f_{s}$ being the sampling interval corresponding to the sampling rate $f_{s}$, their scalar product is defined by ${ }^{7}$

$$
\langle h, q\rangle\left(\tau_{k}\right)=2 \sum_{m=0}^{N-1}\left[H_{m} Q_{m}^{*}+H_{m}^{*} Q_{m}\right] e^{-2 \pi i m k / N} \frac{\Delta_{f}}{S_{h}\left(f_{m}\right)},
$$

where $\Delta_{f}=f_{s} / N, f_{m}=m \Delta_{f}, \tau_{k}=k \Delta$ is the lag of the template-a measure of the relative time shift between the template and signal, $H_{m}=\Delta \sum_{k=0}^{N-1} h_{k} e^{2 \pi i m k / N}$, is the discrete Fourier transform of $h(t)$ (similarly, $Q_{m}$ ), and $S_{h}\left(f_{m}\right)$ is the one-sided noise power spectral density of a detector. In comparing two waveforms the overall amplitude is of no interest and we should, therefore, consider waveforms with unit norm, namely, $\hat{h}=h / \sqrt{\langle h, h\rangle}$. Consequently, the relevant quantity is the scalar product between normalized waveforms defined by

\footnotetext{
${ }^{7}$ It is conventional to define the scalar product in the continuum limit. Here, however, we have given the definition for discretely sampled data, and this is the expression that is used in computing the overlaps.
} 


$$
\mathcal{O}[h, q]=\langle\hat{h}, \hat{q}\rangle .
$$

\section{A. Maximization of the overlaps}

The signal and the template both depend on a set of parameters of the source (e.g., masses and initial spins of the component masses) and its orientation relative to the detector. We shall be concerned with binaries with nonspinning components on quasicircular orbits. Such systems are characterized by two intrinsic parameters, namely, the masses $m_{1}$ and $m_{2}$ of the components, and two extrinsic parameters, namely, the time of coalescence, $t_{C}$, and the phase of the signal at that time, $\phi_{c}$. The overlap integral, therefore, depends on the parameters of the signal and the template, and the relevant quantity is the overlap maximized over these parameters.

The data analysis problem is concerned with digging out a specific signal buried in noisy data. This means that the parameters of the signal are fixed but the data analyst is at liberty to maximize over the parameters of the template. In this paper we will explore the effectualness of templates, that is to say, the overlap maximized over a template's parameters keeping those of the signal fixed. We will do this for several choices of the component masses of the binary. However, the time of coalescence, $t_{C}$, and the phase $\phi_{C}$ of the signal at that time, are arbitrarily chosen to be equal to zero. A caveat is in order concerning the value of the effectualness arising as a result of our choice of $t_{C}$ and $\phi_{C}$ : The maximized overlap is not very sensitive to our choice of $t_{C}$ but it could vary by several percent depending on the choice of a signal's phase, especially when the signal and the template families are not very close in the geometrical sense.

Maximization over a template's masses is carried out using a bank of templates, and the template bank is set up such that, for all signals of the same family as the template, their best overlap with the nearest template is larger than a certain value called the minimal match (MM). Our template placement is as in Ref. [80], which is known to produce, with a probability close to 1 [80], matches larger than the minimal match for the TaylorT1, TaylorT3, TaylorF2, and EOB families of signals (and templates) for the range of masses considered in this paper. We have checked that this is true also for TaylorEt and TaylorT4 families.

We have used a minimal match of $\mathrm{MM}=0.99$ in all cases. Maximization over time of coalescence is accomplished by looking at the overlap integral at different lags $\tau_{k}$. Finally, since our templates are of the form $h_{k}=$ $A_{k} \cos \left(\phi_{k}+\phi_{0}\right)$, where $\phi_{0}$ is an unknown constant phase offset, maximization over $\phi_{0}$ can be achieved by using two quadratures of the template, $h_{k}^{0}=A_{k} \cos \left(\phi_{k}\right)$ and $h_{k}^{\pi / 2}=$ $A_{k} \cos \left(\phi_{k}+\pi / 2\right)$ :

$$
\max _{\phi_{0}} \mathcal{O}[h, q]=\left[\left\langle h^{0}, q\right\rangle^{2}+\left\langle h^{\pi / 2}, q\right\rangle^{2}\right]^{1 / 2} .
$$

When the signal and the template belong to the same family, the maximized overlap is at least MM. When the waveforms belong to different families, the maximized overlap is less than MM.

Our approach here to finding the effectualness of a template with a signal of "fixed" parameters is somewhat different from what is normally followed in the literature, but more appropriate in the context of data analysis. In the literature on the comparisons of different PN models, one normally measures either the best or the minimax overlap [15]. The best overlap gives the maximum of the overlap over the masses and $t_{C}$ but maximized over the constant phases of both the signal and the template. On the other hand, the minimax overlap is the overlap maximized over the masses and $t_{C}$ but minimized over the constant phases of the signal and the template. As mentioned earlier, we fix the phase of the signal to be equal to zero, and hence our effectualness is, in principle, smaller than best overlaps but larger than minimax overlaps. The difference between the best and minimax overlaps is tiny when the effectualness is intrinsically large (i.e., close to 1 ), but could differ by $5 \%$ $8 \%$ when the best overlap is $\sim 0.8$. This should be kept in mind while interpreting our results. Moreover, as mentioned earlier, instead of numerically searching for the maxima of the overlap in the space of masses, we just use a grid of templates with a minimal match of $\mathrm{MM}=$ 0.99 .

We will compute effectualness between every possible template and signal. If our template is the PN approximation $A$ and the signal is the PN approximation $B$, then we are interested in computing the matrix $\epsilon_{A B}$ defined by

$$
\epsilon_{A B} \equiv \max _{\lambda^{A}} \mathcal{O}\left[h_{A}\left(\lambda^{A}\right), h_{B}\left(\lambda^{B}\right)\right],
$$

where $\lambda^{A}$ and $\lambda^{B}$ are the parameters of the template and the signal, respectively. The overlap is symmetric in its arguments $h_{A}$ and $h_{B}$ only if the signal and template, together with their parameters, are interchanged. That is, $\mathcal{O}\left[h_{A}\left(\lambda^{A}\right), h_{B}\left(\lambda^{B}\right)\right]=\mathcal{O}\left[h_{B}\left(\lambda^{B}\right), h_{A}\left(\lambda^{A}\right)\right]$ but, in general, $\mathcal{O}\left[h_{A}\left(\lambda^{A}\right), h_{B}\left(\lambda^{B}\right)\right] \neq \mathcal{O}\left[h_{A}\left(\lambda^{B}\right), h_{B}\left(\lambda^{A}\right)\right]$. Therefore, the maximized overlap $\epsilon_{A B}$ need not be symmetric. The process of maximization, in which the parameters of the "signal" are kept fixed and those of the "template" are varied, breaks down the symmetry. The lack of symmetry arises primarily because the signal manifolds $\mathcal{M}_{A, B}$ representing the two families are distinct; the nearest "distance" from a coordinate point $P$ on $\mathcal{M}_{A}$ to a point on $\mathcal{M}_{B}$ need not be the same as the nearest distance from $P$ on $\mathcal{M}_{B}$ to a point on $\mathcal{M}_{A}$.

\section{B. Effectualness, faithfulness, and loss in event rates}

A direct measure of the efficiency of a template bank is the loss of event rates due to differences between the template family and the exact signal. The loss of event rates is determined by two factors: The effectualness of the templates in matching the exact waveforms and the mini- 
mal match of the template bank itself. In this section, we will quantify this relation.

In Fig. $2^{8}$ we sketch a portion of the waveform space. The solid line represents the template family subspace. Dots represent various waveforms: (i) $h_{\text {Tmplt }}\left(x_{1}\right)$ and $h_{\text {Tmplt }}\left(x_{2}\right)$ are two neighboring templates in the template bank with physical parameters $x_{1}$ and $x_{2}$; (ii) $h_{\text {Tmplt }}\left(x_{0}\right)$ and $h_{\text {Tmplt }}\left(x_{0}^{\prime}\right)$ are waveforms in the same family as the templates to be chosen as discussed below; (iii) $h_{e}\left(x_{0}\right)$, $h_{\mathrm{NR}}\left(x_{0}\right)$, and $h_{\mathrm{EOB}}\left(x_{0}\right)$ are exact, numerical, and $\mathrm{EOB}$ waveforms of the same physical parameters $x_{0}$, respectively. (The EOB waveform is calibrated to the numerical simulation.) We choose $x_{0}^{\prime}$ such that the overlap between $h_{\text {Tmplt }}\left(x_{1}\right)$ and $h_{\text {Tmplt }}\left(x_{0}^{\prime}\right)$ is the minimal match (see below) of the template bank. We choose $x_{0}$ such that $h_{e}\left(x_{0}\right)$ is the exact waveform that has a larger overlap with $h_{\text {Tmplt }}\left(x_{0}^{\prime}\right)$ than with any other waveforms in the template family. This overlap is larger than the one between $h_{e}\left(x_{0}\right)$ and $h_{\text {Tmplt }}\left(x_{0}\right)$ even though they have the same physical parameters, because of the systematic difference between the family of exact waveforms and the family of templates.

We define the distance in the waveform space between two waveforms $h$ and $q$ by the scalar product $\sqrt{1-\mathcal{O}[h, q]}$. For convenience, we define the mismatch to be the square of the distance. The overlap between $h_{\text {Tmplt }}\left(x_{1}\right)$ and $h_{\text {Tmplt }}\left(x_{0}^{\prime}\right)$ is the minimal match, and we denote the corresponding mismatch by $d_{\mathrm{MM}}=1-\mathrm{MM}$. Similarly, $1-d_{\mathrm{E}}$ and $1-d_{\mathrm{F}}$ are the effectualness and faithfulness of the template family with the exact waveform $h_{e}\left(x_{0}\right)$, respectively. The mismatch between $h_{e}\left(x_{0}\right)$ and the closest template $h_{\text {Tmplt }}\left(x_{1}\right)$ quantifies the reduction in the signal-to-noise ratio when the template bank is used to search for the exact waveform. We denote this mismatch by $d_{\text {ER }}$. When these mismatches are small, by Pythagorean theorem, we have an approximate relation $d_{\mathrm{ER}} \simeq d_{\mathrm{MM}}+$ $d_{\mathrm{E}}$. Assuming uniform spatial distribution of sources, the reduction in event rate is $1-\left(1-d_{\mathrm{ER}}\right)^{3} \simeq 3 d_{\mathrm{ER}}$. Therefore, if we want to satisfy the usual requirement of $<10 \%$ loss in event rate, we need $d_{\mathrm{ER}}=d_{\mathrm{MM}}+d_{\mathrm{E}}<3.5 \%$. Typical minimal match adopted in current searches has either $d_{\mathrm{MM}}=3 \%$ or $d_{\mathrm{MM}}=1 \%$, which means, in the first case, an extremely rigorous requirement on the effectualness $\left(d_{\mathrm{E}}<0.5 \%\right)$, or in the second case, a reasonable requirement of $d_{\mathrm{E}}<2.5 \%$. The latter is achievable by PN models. Note that, if both the minimal match of a template bank and the effectualness of the template model are $97 \%$, the loss in event rate rises to $17 \%$.

However, it is not possible to calculate $d_{\mathrm{ER}}$ since we do not know the exact waveform $h_{e}\left(x_{0}\right)$. In this paper, we adopt two strategies to estimate $d_{\mathrm{ER}}$ : (i) we calculate the mutual effectualness of PN models for low-mass binaries and assume it to be a good representation of their effec-

${ }^{8}$ This figure is very similar to Fig. 3 of Ref. [26].

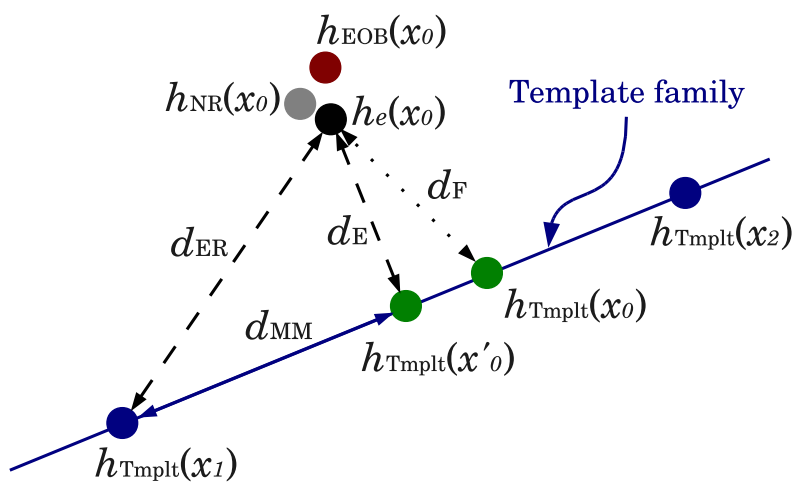

FIG. 2 (color online). Schematic plot of the distance (or mismatch) relation between templates and exact, numerical, and EOB waveforms.

tualness with exact waveforms; (ii) we approximate $h_{e}\left(x_{0}\right)$ with the EOB waveform $h_{\mathrm{EOB}}\left(x_{0}\right)$ calibrated to the numerical simulations. We can verify the goodness of the latter assumption as follows. The mismatch between the best EOB waveforms $[45,46]$ and the numerical waveforms is less than $10^{-3}$. In Ref. [46], the authors calculated the mismatch among accurate numerical waveforms generated by simulations with different resolutions and/or extraction schemes as well. They found that the mismatch is less than $10^{-4}$. We consider the latter as an estimate of the mismatch between exact and numerical waveforms. In the worst case, the mismatch between the exact and EOB waveforms with the same physical parameters is roughly $\left(\sqrt{10^{-3}}+\right.$ $\left.\sqrt{10^{-4}}\right)^{2}=1.7 \times 10^{-3}$. Therefore, we can conclude that by approximating $h_{e}\left(x_{0}\right)$ with $h_{\mathrm{EOB}}\left(x_{0}\right)$, we underestimate the loss of event rate by at most $0.5 \%$.

Notice that the effectualness result presented in the following sections is slightly different from $1-d_{\mathrm{E}}$. It is obtained through discrete searches over template parameters using template banks with $\mathrm{MM}=0.99$ rather than through continuous searches. Therefore, the mismatch associated with this effectualness result includes already the discreteness effect in the template banks, i.e. a mismatch $d_{\mathrm{MM}}^{(0)}=0.01$. In this case, if a search is carried out with a template bank of a different minimal match, say $\mathrm{MM}=$ $1-d_{\mathrm{MM}}=0.97$, to calculate the loss of event rate, a mismatch of $d_{\mathrm{MM}}-d_{\mathrm{MM}}^{(0)}=0.02$, instead of $d_{\mathrm{MM}}$, needs to be added to the effectualness result in this paper, i.e. $d_{\mathrm{ER}}=d_{\mathrm{MM}}-d_{\mathrm{MM}}^{(0)}+d_{\mathrm{E}}$. The only exception in this paper is the effectualness result between EOB models presented in the Conclusions which is obtained through a continuous search.

\section{Choice of binary systems and PN orders}

We have chosen three conventional systems, binary neutron stars (BNS), binary black holes (BBH), and binary neutron-star-black-hole systems. We have chosen the BNS 
and $\mathrm{BBH}$ systems to be slightly asymmetric, $(1.38,1.42) M_{\odot}$ and $(9.5,10.5) M_{\odot}$, but the NS-BH system is chosen to be the conventional $(10,1.4) M_{\odot}$ system. To this we have added another binary with component masses $(4.8,5.2)$ which lies on the border line between where most PN families are similar to one another and where they begin to differ.

We compute overlaps maximized over a template bank between seven different models (TaylorT1, TaylorT2, TaylorT3, TaylorT4, TaylorF2, TaylorEt, EOB), each at three different PN orders $\left(v^{4}, v^{6}, v^{7}\right)$. The results will be presented in the form of a set of figures. For each mass pair there will be one figure consisting of nine panels (one panel for each PN order), each panel containing seven curves (one each for each template family at that order) and each curve with 21 data points corresponding to signals from the seven PN families at each of three different PN orders, 2PN, 3PN, and 3.5PN.

\section{RESULTS OF THE EFFECTUALNESS OF PN TEMPLATES}

We will present the results of our investigation in two complementary ways. We will first discuss the effectualness of the different PN families with each other. Such an analysis will help us understand how "close" the various families of PN approximants are at different PN orders in regard to the construction of detection templates. We then go on to look at the effectualness of the different approximants with the EOB signal that contains not only the inspiral but also the merger and ringdown parts. The goal of the latter analysis is to identify the region in the parameter space where one can safely use any $\mathrm{PN}$ approximant template in a search, without worrying about the loss in signal-to-noise ratio that might arise due to our lack of knowledge of the real signal, but without expending undue computational resources. Outside this region, however, one must use template families that are calibrated to waveforms obtained from numerical-relativity simulations.

\section{A. Mutual effectualness of various PN inspiral template banks}

The effectualness of the different PN families with each other is shown in Fig. 3 (Initial LIGO) and Fig. 4 (Advanced LIGO) for four different systems with component masses as indicated at the top of each subfigure. In each subfigure, the top panels correspond to the effectualness of different template families at 3.5PN order, middle panels to 3PN order, and bottom panels to $2 \mathrm{PN}$ order. For each template family considered we find their overlap with signals from different PN orders (as indicated along the $x$ axis) and approximants (as indicated by the text T1, T2, etc.). Each symbol corresponds to the overlap obtained by a different template family: (black) circles to TaylorT1, (red) squares to TaylorT2, etc., with signals from different
PN families. Note that we have used the logit scale for the vertical axis. This is so that (minor) disagreements between the different approximants are made clearly visible. Note that since we are considering systems with low total mass, say $\leq 20 M_{\odot}$, in this section we use the EOB model terminated at the EOB light ring; that is, we do not include the merger and ringdown parts.

Conventionally, one says that two approximants $A$ and $B$ are in close agreement with each other if their mutual effectualness $\epsilon_{A B}$ is 0.965 or greater [18]. Though we shall mildly relax this target a bit to 0.95 for ease of presentation, we shall also indicate in Sec. VII the region of the parameter space where the effectualness is better than 0.965 , but we shall also quote regions where the effectualness drops to a low value of 0.9 . The latter should be helpful for data analysis pipelines that employ a multistage hierarchical search, the first stage of which deploys a coarse grid of templates.

These figures reveal many different aspects of the (dis) agreements between the different approximants at PN orders 2PN, 3PN, and 3.5PN, and we shall principally highlight in our discussion the "diagonal" behavior, i.e. overlaps of each template family with a signal family from the same PN order. Focusing first on the Initial LIGO results (Fig. 3), we see the evidence for the clustering of the various approximants at $3 \mathrm{PN}$ and $3.5 \mathrm{PN}$ orders for systems with a smaller total mass. In the case of BNS with component masses $(1.38,1.42) M_{\odot}, 2 \mathrm{PN}$ diagonal overlaps are dispersed between 0.74 and 1 , and 3PN and 3.5PN overlaps are all above 0.95 , with TaylorEt having the smallest overlaps.

In the case of $\mathrm{BBH}$ with component masses $(4.8,5.2) M_{\odot}, 2 \mathrm{PN}$ overlaps are between 0.8 and $1,3 \mathrm{PN}$ overlaps are all greater than 0.95 except TaylorEt, and 3.5PN overlaps are greater than 0.95 for all except TaylorEt, TaylorT3, and EOB. There are several important points to note: As discussed in Sec. IV, TaylorT3 terminates somewhat prematurely before reaching the last stable orbit. Therefore, one expects to have poorer overlaps for all templates if the TaylorT3 signal terminates in band, which will be the case for systems with a total mass greater than about $10 M_{\odot}$. The asymmetry in the overlaps mentioned in Sec. VA is apparent in the case of TaylorEt: The overlaps of all templates with the TaylorEt signal are greater than the converse, namely, the overlaps of the TaylorEt templates with other signals. The smaller overlaps of the EOB templates (terminated at the light ring) are not surprising, given the very different termination frequencies of the two families and the fact that the EOB waveform has power in the band beyond the last stable orbit.

In the case of NS-BH with component masses $(1.4,10) M_{\odot}, 2 \mathrm{PN}$ diagonal overlaps are distributed between 0.6 and 1 , and 3PN and 3.5PN overlaps are consis-

\footnotetext{
${ }^{9} \operatorname{Recall} \operatorname{logit}(p)=\log \left(\frac{p}{1-p}\right)$.
} 

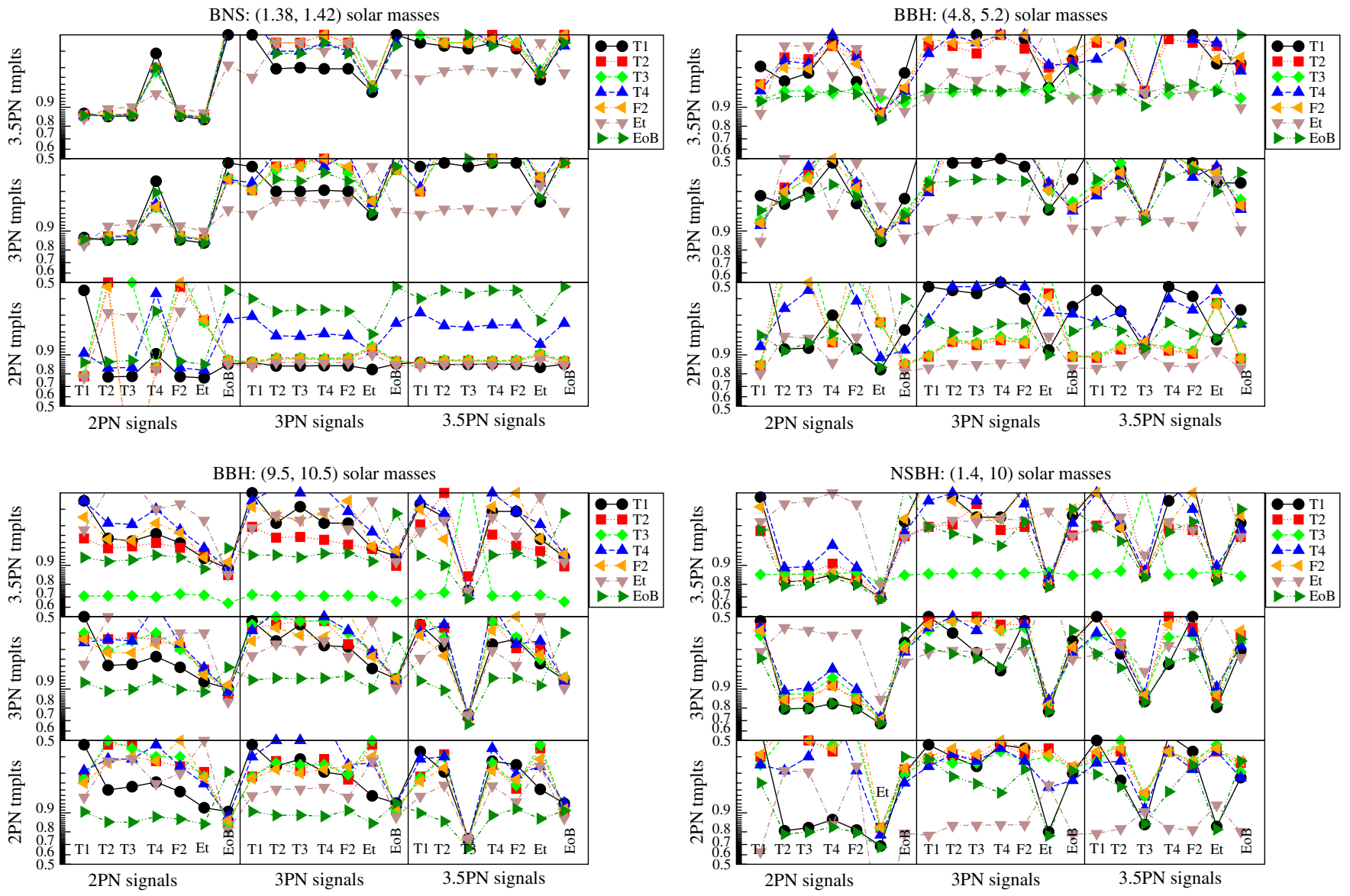

FIG. 3 (color online). The plot shows the effectualness of templates and signals of different post-Newtonian families and orders for four different binary systems for Initial LIGO. For a template from a given PN approximation (indicated by different line styles and symbols) and order (top panel 3.5PN, middle panel 3PN, and bottom panel 2PN), we compute the effectualness of each of the templates with signals from each of the seven families, TaylorT1 (T1), TaylorT2 (T2), TaylorT3 (T3), TaylorT4 (T4), TaylorF2 (F2), TaylorEt (Et), and EOB, at 2PN, 3PN, and 3.5PN orders. For instance, solid lines with filled circles give the effectualness of TaylorT1 templates at 3.5PN (top panel), 3PN (middle panel), and 2PN (bottom panel) orders, with signals that belong to different PN approximations and orders. Clockwise, the panels from top left correspond to binaries consisting of two neutron stars with masses $1.38 M_{\odot}$ and $1.42 M_{\odot}$, two black holes with masses $4.8 M_{\odot}$ and $5.2 M_{\odot}$, two black holes with masses $9.5 M_{\odot}$ and $10.5 M_{\odot}$ and, finally, a neutron star and a black-hole binary with component masses $1.4 M_{\odot}$ and $10 M_{\odot}$.

tently above 0.95 except for TaylorEt signals (both orders) and TaylorT3 (at 3.5PN).

In the case of $\mathrm{BBH}$ with component masses $(9.5,10.5) M_{\odot}$, there is no agreement between approximants irrespective of the PN order. In this sense, one cannot trust using any particular approximant as a search template.

Let us now turn to Fig. 4, which depicts the results for Advanced LIGO noise power spectral density. In the case of BNS with component masses $(1.38,1.42) M_{\odot}$, the $2 \mathrm{PN}$ diagonal overlaps are between 0.4 and 1 (note that some of the data points are below the scale of 0.5 that we employ). The 3PN (except the TaylorEt signal) and 3.5PN (except the TaylorT3 template and TaylorT3 and TaylorEt signals) overlaps are uniformly larger than 0.95 . The effectualness of all templates with the TaylorEt signal is generally smaller (0.6-0.8) than the effectualness with a TaylorEt template. In the case of $\mathrm{BBH}$ with component masses
$(4.8,5.2) M_{\odot}$, the $2 \mathrm{PN}$ overlaps could be as small as 0.65 . For all approximants at 3PN order (except TaylorT3 templates) and 3.5PN order (except TaylorEt and TaylorT3 templates) the overlaps are 0.95 or greater. In the case of NS-BH with component masses $(1.4,10) M_{\odot}$, the $2 \mathrm{PN}$ overlaps are as low as 0.4 . At 3PN and 3.5PN, the overlaps are larger than 0.95 except in the case of TaylorEt signals (3PN, 3.5PN) and TaylorT3 templates (3.5PN). In the case of $\mathrm{BBH}$ with component masses $(9.5,10.5) M_{\odot}$, the $2 \mathrm{PN}$ overlap could be as low as 0.7 . The overlaps are larger than 0.95 at 3PN except in the case of EOB templates and TaylorEt and EOB signals. Finally, at 3.5PN order the different approximants are seen not to agree with each other very well. The cause of these features is the same as our discussion for Initial LIGO.

We conclude with some brief remarks on the "nondiagonal" cases displayed in the two figures. The asymmetric 

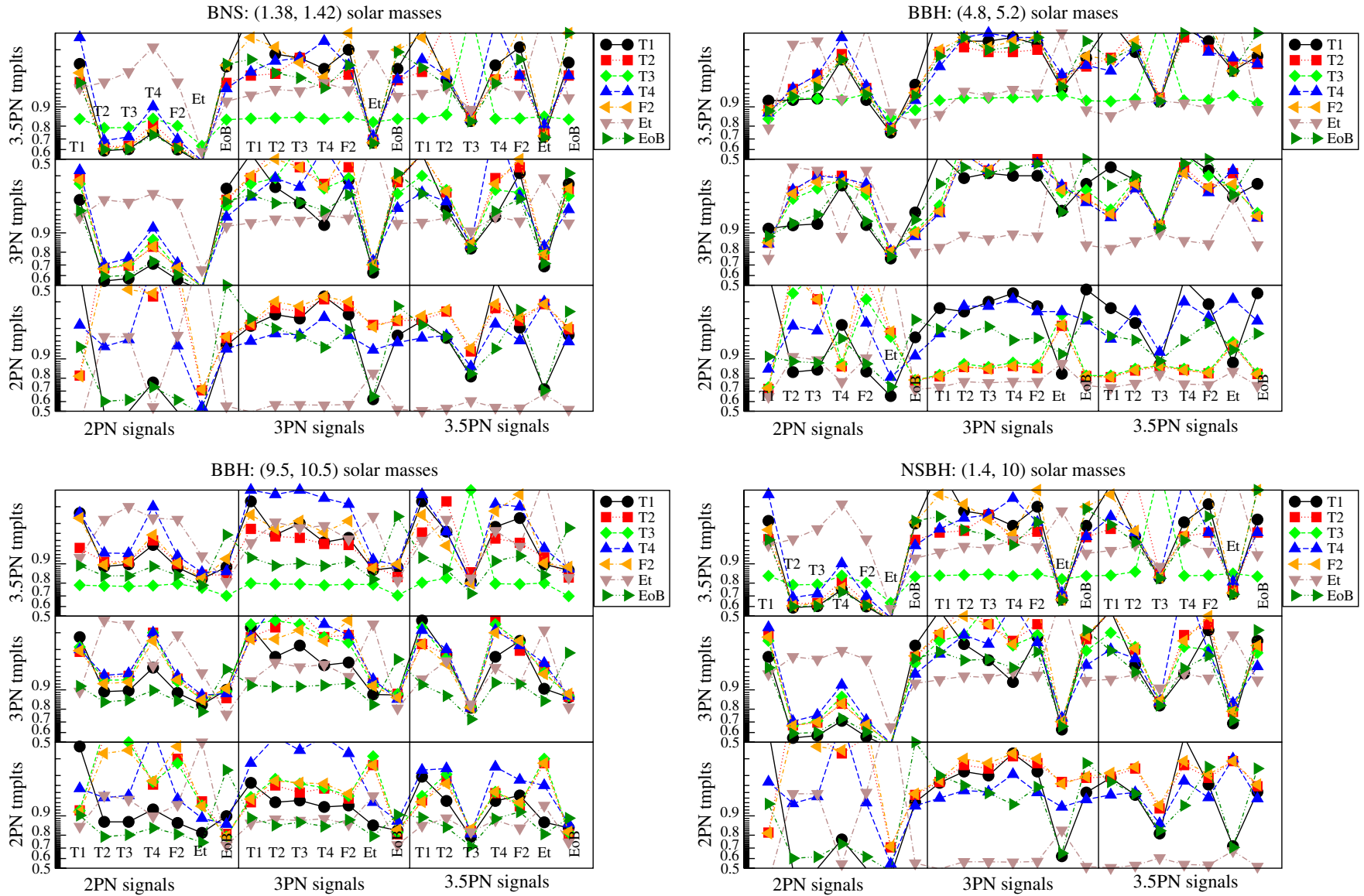

FIG. 4 (color online). Same as Fig. 3 but for Advanced LIGO.

roles of signal and template arising from the maximization is obvious from the different panels. From the panels for 2PN signals and 3PN (3.5PN) templates for systems involving neutron stars, it is interesting to see that higher PN order approximant templates do not necessarily lead to higher effectualness. One can also read off whether 3PN templates are as effective as the 3.5PN templates for various systems and various detectors. The figures condense a variety of such insights and may be useful to look at specific issues when required.

\section{B. Discussion}

In the case of binary neutron stars, the merger occurs far outside the sensitive band of the detector, and even the late stages of inspiral are out of band. Binary neutron stars will very much be in the adiabatic regime as the signal sweeps through the band, and a good test of the PN approximation is to ask how well the different waveforms agree with one another in this regime. The finite bandwidth of the detector essentially probes this regime for binary neutron stars. Note that the effectualness amongst different PN families at $2 \mathrm{PN}$ order is pretty poor but greater than 0.95 (with the exceptions discussed earlier) at 3PN and 3.5PN orders. In the case of Advanced LIGO (cf. Fig. 4), the lower fre- quency cutoff used in computing the overlap integrals is $20 \mathrm{~Hz}$, and a binary neutron star spends more than 750 cycles in band. Effectualness of 0.95 or greater means that the waveforms remain in phase over the entire duration of the signal. Of course, in reality the parameters of the signal and the template are not the same, but even so this is a remarkable success of the PN scheme.

For a BBH system with masses $(4.8,5.2) M_{\odot}$, we see that $2 \mathrm{PN}$ and $3 \mathrm{PN}$ order templates are qualitatively similar to the binary neutron star case. However, we can see a marked deterioration of the effectualness at 3.5PN order. For a system of total mass of $10 M_{\odot}$, the Schwarzschild LSO occurs at $\sim 440 \mathrm{~Hz}$ and the detector is sensitive to the late stages of the inspiral phase. It is not entirely surprising, therefore, that different PN orders do not agree with each other to the same extent as in the binary neutron star case. However, note that, with the exception of TaylorT3, which terminates at a frequency somewhat lower than others, and TaylorEt, all other templates have effectualness of 0.95 or better with each other. Among approximants that agree with each other, EOB has the smallest effectualness. This is because the latter model contains the plunge phase of the coalescence with ending frequencies far higher than the LSO, while other approximants do not have the plunge phase. 
The LSO of a BBH with component masses $(9.5,10.5) M_{\odot}$, is at $\sim 220 \mathrm{~Hz}$ and the plunge phase spans $220 \mathrm{~Hz}$ to about $600 \mathrm{~Hz}$. Therefore, the detector is pretty sensitive to the late phases of the coalescence. We see deterioration of the effectualness, both at 3PN and 3.5PN orders. Apart from TaylorT3, whose poor overlaps at 3.5PN are explained by the early termination of the signal, the EOB stands out by achieving overlaps as low as 0.92 with other families.

As a final example, for the effectualness of templates for a signal from a neutron-star-black-hole binary of masses $(1.4,10) M_{\odot}$, we see that the different PN families, including the EOB, are in good agreement with each other, with the sole exception of TaylorEt. In fact, the closeness amongst different families seems to be somewhat better than the BBH system of component masses $(9.5,10.5) M_{\odot}$.

At this juncture, it is worth pointing out that our numerical results for effectualness in the subset of cases where TaylorEt is chosen as the signal model are consistent with those in Ref. [48], which investigated the fitting factors to ascertain if 3.5PN TaylorEt signals could be effectually and faithfully searched by TaylorT1, TaylorT4, and TaylorF2 templates. There is agreement too on the general features of our results with regard to systematic biases, the dependence on the total mass and qualitative factors underlying them. However, this agreement of numerical results for faithfulness and effectualness in no way extends to the general motivation and claims regarding the TaylorEt approximants [48,49,51], and, hence, these claims are worth clarifying.

Indeed, there is no basis to refer to the $x$-based orbital phasing equation (3.5a) as Newtonian [51], since the $\omega$ here is $n \mathrm{PN}$ accurate (depending on the PN-generation order one is working at) and implicitly incorporates conservative contributions to gravitational-wave phase evolution at various PN orders. It is incorrect to claim [48] that conservative contributions to the gravitational-wave phase evolution do not appear in the standard approximants, or that the TaylorEt-based scheme treats conservative and radiation-reaction contributions more equitably than the standard $x$-based approximants. It is misleading [48] to refer to only TaylorEt-based approximants as "fully gauge invariant in contrast to EOB" (especially in the circular orbit case). All $x$-based schemes are also fully gauge invariant. Finally, one may work in specific convenient coordinate systems as do EOB and numerical-relativity simulations, as long as one deals with and compares gauge invariant quantities at the end.

In our view, the very different behavior of the TaylorEt approximant relative to the standard $x$-based approximants may be traced to the manner in which the orbital phasing is "packaged" in the two schemes. In the $x$-based schemes the orbital phasing is implicitly in a resummed form, since the phasing is written in an appropriate $\mathrm{PN}$-accurate angular velocity $\omega_{n \mathrm{PN}}(n=2,3$ for $2 \mathrm{PN}, 3 \mathrm{PN}$ templates). On the other hand, the representation in terms of $\zeta$, relative to the $x$ schemes, is a reexpanded form. And indeed, based on the comparison between analytical schemes and numerical-relativity simulations, the $\zeta$ schemes do relatively worse. The feature related to the monotonic convergence of the TaylorEt scheme is of secondary importance in comparison to the main requirement of high phasing accuracy of an analytical model with numerical-relativity simulations over all mass ratios.

A few general comments are in order before we conclude this section. We do not believe that at present there are convincing theoretical reasons to consider any one particular PN family of inspiral models to be a privileged signal model. Consequently, the best that one can do is to examine the mutual closeness of these various inspiral models, as we have done, and work at the PN order where these various template families display the greatest agreement. It is precisely in this regard that the viewpoint we present here differs from those in $[48,49,51]$ which assume primacy for one specific approximant, namely, the TaylorEt approximant, based on theoretical motivations that at present do not appear to be fundamental or compelling. Consequently, though there is no difference in the numerical results in the subset of cases that are common in our investigations, there is a big difference in the conclusions that we believe can be inferred. For instance, before one can legitimately decide on the inability of standard template banks in the gravitational data pipeline to detect signals from binaries with eccentricity [51], it is necessary to first fold in the differences in the simpler quasicircular case arising on account of different parametrizations. Similar considerations should be borne in mind when dealing with analogous problems in the spinning case.

Based on the analysis presented heretofore, we conclude that the mutual effectualness of different families of PN approximants are close to each other (i.e. more strongly clustered) at 3PN and 3.5PN orders, ${ }^{10}$ as long as the total mass is less than about $12 M_{\odot}$ (with the exceptions discussed in the previous section).

For heavier binaries, the approximants begin to differ considerably, and this is almost entirely because the adiabatic approximation begins to breakdown and the plunge and the merger phases become more and more important. Hence, in the next section we will supplement the present analysis by looking more precisely into the overlaps of the different PN templates with a prototype of the more com-

\footnotetext{
${ }^{10} \mathrm{We}$ find that the overlaps of the full EOB model obtained with the Virgo design PSD are very similar to those obtained for Advanced LIGO PSD for all Taylor models. The only differences are in the case of the EOB model cutoff at the light ring, where overlaps obtained with the Virgo PSD are smaller by a few percent. Needless to say, the situation for a space detector like LISA can be expected to be even more different and interesting to study.
} 
plete signal model, namely, the EOB model, including the merger and ringdown parts.

\section{Biases in the estimation of parameters}

Recall that, in the computation of the effectualness, one maximizes the scalar product of a (normalized) signal with a template over the parameters of the template while keeping the parameters of the signal fixed. Therefore, one can get an idea of how dissimilar the parameters of an approximant need to be in order to match a given signal. This is a systematic effect that leads to a bias in the estimation of parameters if the template approximant is not the same as the signal approximant. Let the total mass of the signal and template waveforms be, respectively, $M_{\mathrm{Sgnl}}$ and $M_{\text {Tmplt }}$, when the scalar product is maximized. The percentage bias $\Delta M$ in the total mass is defined as $\Delta M=100(1-$ $M_{\text {Tmplt }} / M_{\text {Sgnl }}$, and similarly for the symmetric mass ratio $\nu$.

For a given binary, the biases are qualitatively similar for Initial and Advanced LIGO noise power spectral densities. In general, the biases are appreciably smaller at $3 \mathrm{PN}$ and 3.5PN orders than at $2 \mathrm{PN}$ order and progressively increase with the total mass, although they are far larger than the statistical errors computed using the Fisher information matrix [24]. Figure 5 plots the percentage biases in the total mass $M$ and symmetric mass ratio $\nu$ at 3.5PN order. The left two (right two) columns use the Initial LIGO
(Advanced LIGO) noise spectral density. For the four systems considered, namely, $(1.38,1.42) M_{\odot},(4.5,5.2) M_{\odot}$, $(1.4,10) M_{\odot}$, and $(9.5,10.5) M_{\odot}$ binaries, the largest bias in the total mass $M$ is $1 \%, 20 \%, 20 \%$, and $20 \%$, respectively, and the symmetric mass ratio $\nu$ is $1 \%, 25 \%, 70 \%$, and $25 \%$, respectively.

\section{RESULTS OF THE EFFECTUALNESS OF PN TEMPLATES WITH THE FULL WAVEFORM}

Having established the mutual closeness of the different families of PN approximants at 3PN and 3.5PN orders (for determining effectual templates for detection) in the regime where the approximation is expected to be valid, let us now examine the region in the parameter space where PN families can be used as search templates. To achieve this goal we will use the EOB model calibrated to numerical-relativity simulations [40]. For brevity, we have omitted plots of the effectualness of the 3PN approximants with this EOB model; they are quite similar to the 3.5PN plots.

Although Ref. [40] explored the agreement between the EOB model and numerical simulations for several modes, in this study we will work with only the dominant harmonic (i.e., the $h_{22}$ mode) at leading PN order. Higherorder amplitude corrections are known to be important for parameter estimation [61,62], and a future study must repeat this investigation with the full waveforms.
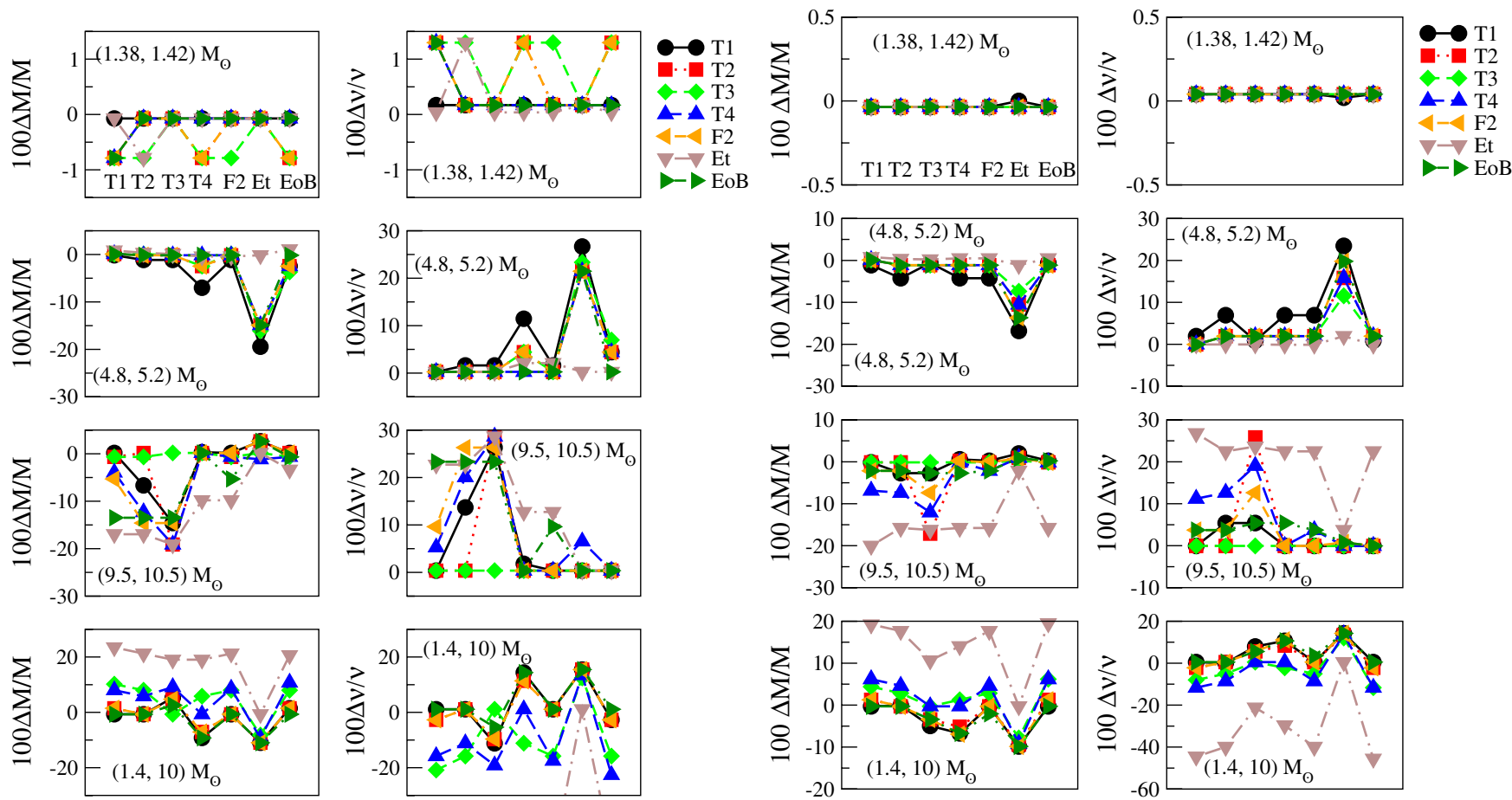

FIG. 5 (color online). Percentage bias in the estimation of the total mass $M$ and symmetric mass ratio $\nu$ at $3.5 \mathrm{PN}$ order. The left two columns are for Initial LIGO and the right two are for Advanced LIGO. The bias $\Delta M / M$ is defined as $\Delta M=\left(1-M_{\text {Tmplt }} / M_{\text {Sgnl }}\right)$, where $M_{\text {Sgnl }}$ and $M_{\text {Tmplt }}$ denote the total mass corresponding to the signal and the template that obtained the maximum effectualness, respectively (and similarly for $\nu$ ). What is plotted is the percentage bias. The bias arises because the template family (as indicated in the key) is different from the signal family (as indicated in the top left panel as T1, T2, etc.). 

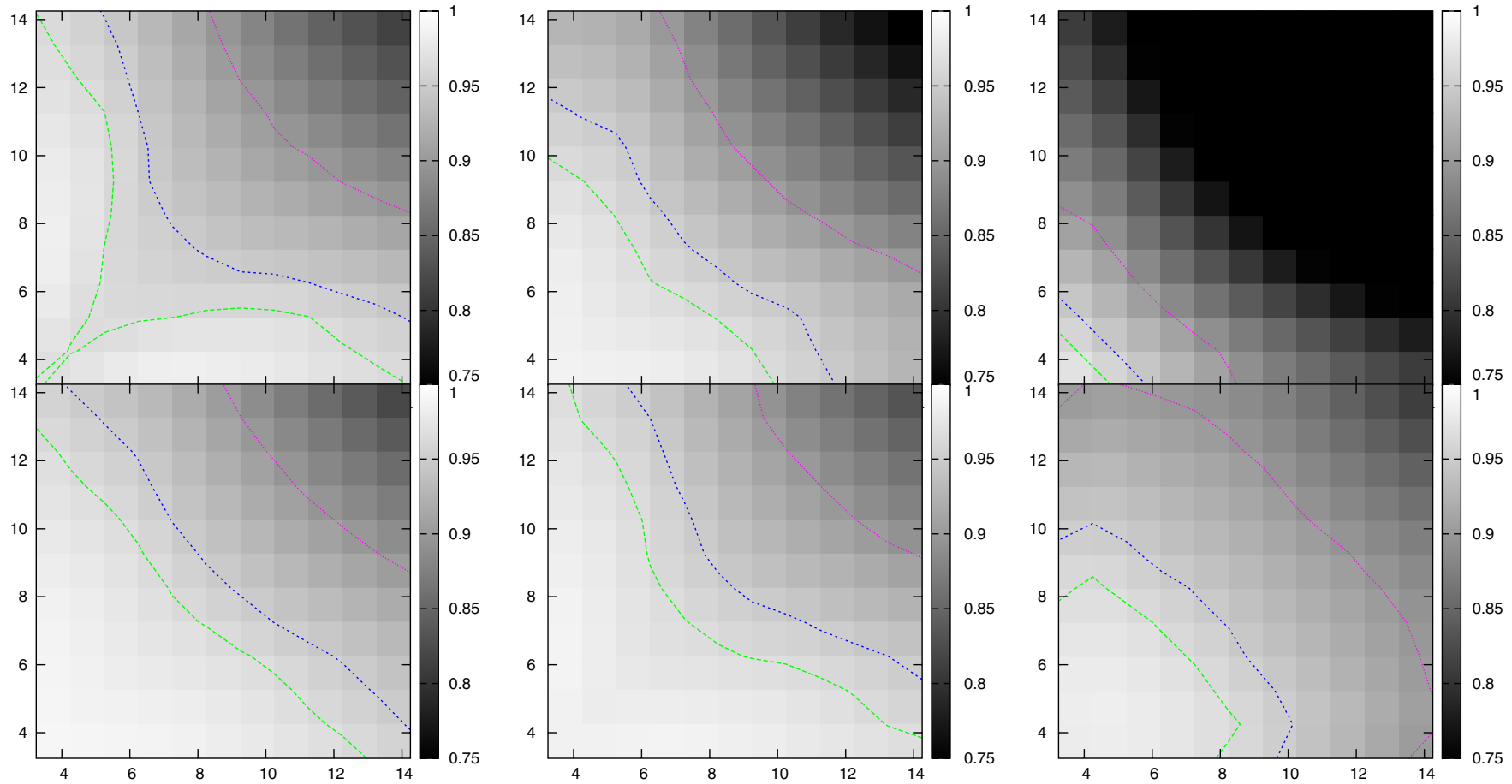

FIG. 6 (color online). Overlaps of different 3.5PN approximants with the EOB inspiral-merger-ringdown signal in Initial LIGO in the $\left(m_{1}-m_{2}\right) M_{\odot}$ plane. The approximants considered from left to right are TaylorT1, TaylorT2, TaylorT3 (top panels), and TaylorT4, TaylorF2, TaylorEt (bottom panels). The contours correspond to overlaps of 0.9, 0.95, and 0.965.

Figure 6 shows the effectualness of the six PN families TaylorT1, TaylorT2, TaylorT3 (top panels, respectively, from left to right), TaylorT4, TaylorF2, and TaylorEt (bottom panels, respectively, from left to right) for Initial LIGO

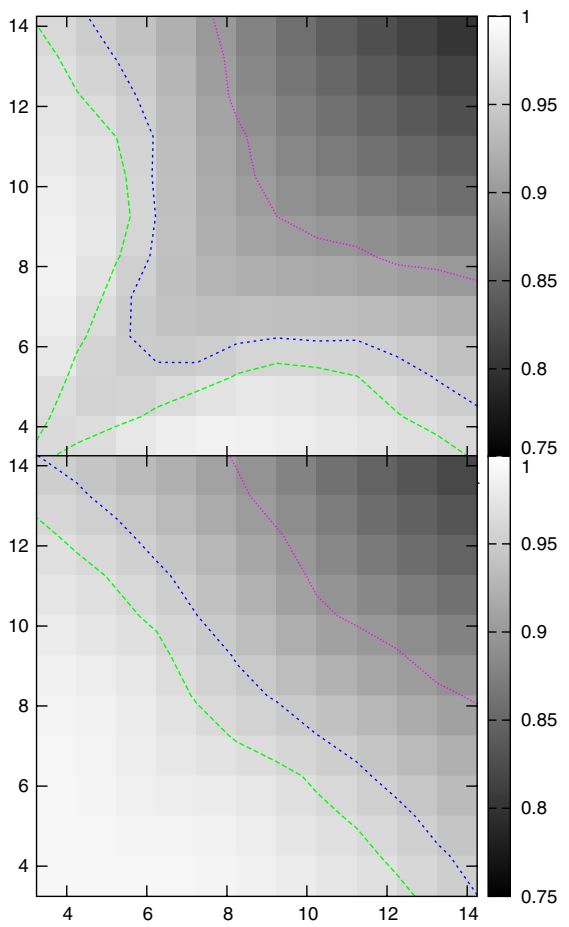

noise power spectral density. Figure 7 shows the same but for Advanced LIGO noise power spectral density. The effectualness was computed using a hexagonal template bank [80] and is shown as a gray-scale map in the space of
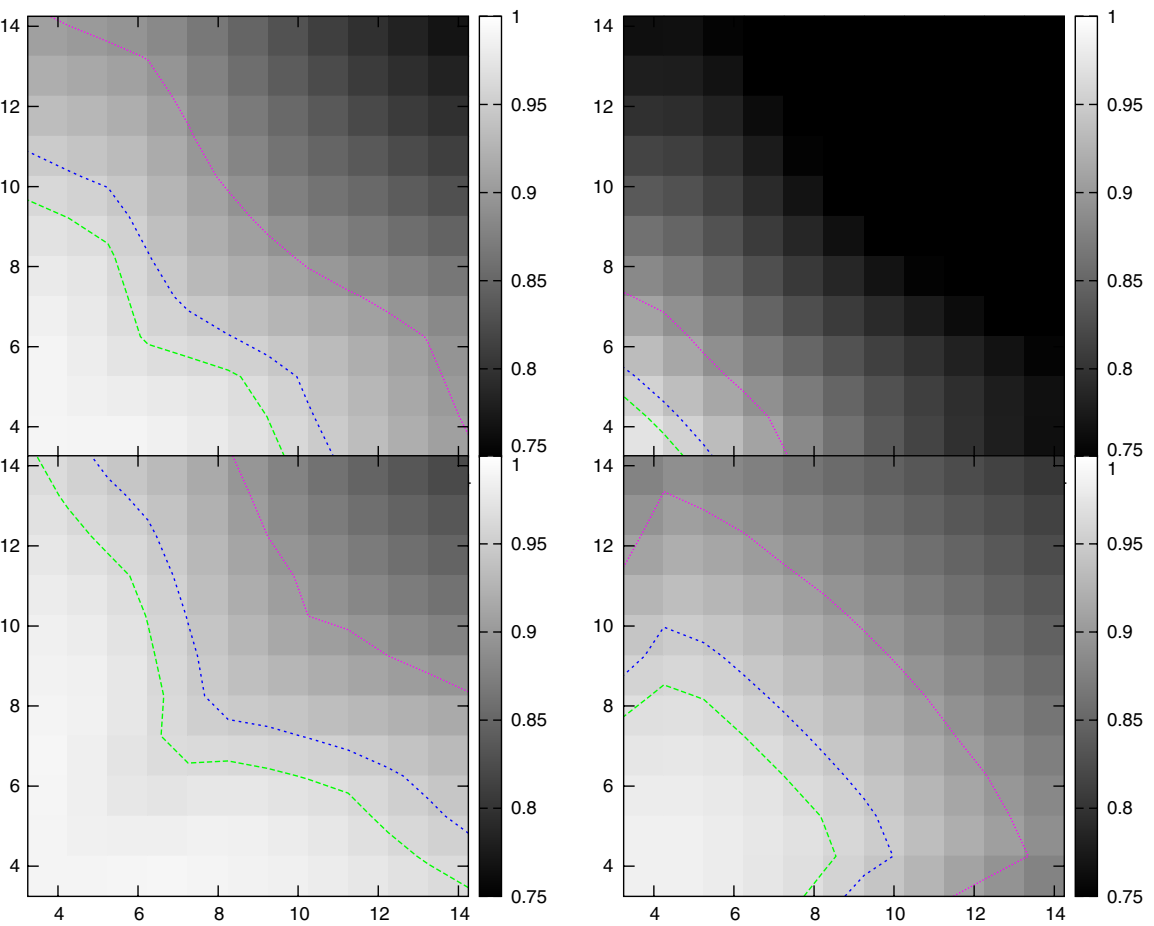

FIG. 7 (color online). Same as Fig. 6 except that the noise spectral density is that of Advanced LIGO. The contours correspond to overlaps of $0.9,0.95$, and 0.965 . 
the component masses that are taken to vary from $3 M_{\odot}$ to $14.5 M_{\odot}$. For all the maps, we have chosen the gray scale to vary from 0.76 to 1 . The dotted contours show effectualness at three values: $0.965,0.95$, and 0.90 .

The trends of the overlaps are rather similar irrespective of which noise power spectral density we use, although the actual overlaps are systematically smaller in the case of Advanced LIGO as compared to Initial LIGO. This is due to the broader frequency sensitivity of the former in relation to the latter. The following discussion is, therefore, applicable in both cases.

Let us first note some peculiarities. TaylorT3 at 3.5PN leads to particularly ineffectual templates. As mentioned before, TaylorT3 at 3.5PN terminates rather prematurely. The LSO defined by the Schwarzschild potential is at $f_{\text {LSO }} \sim\left(440 / 10 M_{\odot}\right) \mathrm{Hz}$, but TaylorT3 approximants at $3.5 \mathrm{PN}$ terminate at $\sim\left(220 / 10 M_{\odot}\right) \mathrm{Hz}$. This discrepancy is so large that even with the biases in the component masses allowed in the computation of the effectualness (recall that we maximize the overlap over template masses), which, in principle, makes it possible for a template of a lower mass to match a signal of a higher mass, TaylorT3 is unable to achieve good overlaps. This is because a mismatch in the component masses can make a template more, or less, asymmetric than the signal, which has the effect of increasing, or decreasing, the duration of the template relative to the signal. While small differences in the ending frequencies can be achieved by a mismatch in the total mass without affecting the signal duration too greatly, large differences cannot be compensated by such a mismatch in the parameters.

At 3PN and 3.5PN the effectualness of TaylorEt with an EOB signal for a binary of component masses $(3,10) M_{\odot}$ [respectively, $(10,10) M_{\odot}$ ] is 0.83 and 0.90 [respectively, 0.87 and 0.89 ]. This is because amongst all PN approximants TaylorEt seems to converge far slower than any other. Further, an examination of the coefficients in the PN terms of the phasing formulas in Eqs. (3.13) indicates that higherorder PN terms have increasingly greater coefficients. In general, it has been observed that the appearance of such larger coefficients in higher-order terms of an approximant scheme inevitably worsens its convergence, and the present instance may be no exception to this case. ${ }^{11}$

A final observation: Some of the models, TaylorT1 in particular, have lower overlaps along the $m_{1}=m_{2}$ or $\nu=$ $1 / 4$ line. A similar feature has been observed in other cases in the past [36] and can be traced to more limited possibilities in maximization over $\nu$ in the equal-mass case. For unequal-mass systems one can explore template $\nu$ values both smaller and larger than the signal $\nu$ values. Certain template families might agree better with the "exact" signal only for template $\nu$ values larger than the signal $\nu$

\footnotetext{
${ }^{11}$ While comparing the coefficients it may be useful to note that $v \simeq 1 / \sqrt{6}$ corresponds to $\zeta$ in the range of $0.136-0.138$ depending on the symmetric mass ratio $\nu$ and the PN order.
}

value. However, for equal-mass configurations we are already on the boundary $\nu=1 / 4$, and if we are constrained to only physical values of template $\nu$, one can only admit values of $\nu$ smaller than $1 / 4$.

With the exception of the peculiarities noted above, we see that all approximants do progressively better at higher PN orders. Conclusions drawn in the previous section with regard to the mutual closeness of the different families of PN approximants are further corroborated here, where we have measured the overlaps with a signal that is matched to numerical-relativity simulation, which can, therefore, be taken to be close to what a real signal might be.

Computationally, TaylorF2, with its phasing formulas given explicitly in the Fourier domain, is the least expensive. This is because matched filtering is most easily carried out in the Fourier domain, which means that a timedomain approximant must be Fourier transformed before computing the cross correlation. By employing TaylorF2 models one can avoid one forward Fourier transform. Moreover, TaylorF2 offers the flexibility in the choice of the ending frequency. Unlike the time-domain models, which have a natural ending frequency defined by the extremum of the binding energy or the frequency (before reaching the LSO) at which the evolution stops, TaylorF2 has no such restriction. In fact, as obtained in Refs. [36,37], by extending the upper cutoff beyond the usual upper cutoff (i.e., the Schwarzschild LSO), the TaylorF2 model matches remarkably well with numerical-relativity waveforms for a far greater range of masses. However, as noted in Ref. [37] the ending frequency that must be employed in order to achieve the best match with numerical-relativity waveforms depends on the noise power spectral density. This could turn out to be an unnecessary computational burden in a data analysis pipeline. The alternative is to choose the upper frequency cutoff as an additional search parameter or allow unphysical values of $\nu>0.25$ $[36,37,48]$, or to include a p4PN term in the template phase and calibrate it to numerical simulations [36]. The first two choices would result in an unwarranted increase in the computational cost of a search, as also in the false alarm rate, and we advise against it. The third choice could be pursued, but it should be augmented by a more complete description of the merger/ringdown signal-for example, by introducing a slope break in the waveform amplitude and a superposition of Lorentzians [35,36]

If a search requires the minimal match to be much smaller than 0.95 (as, for example, in a hierarchical search), one can extend a search with TaylorF2 to a total mass of $20 M_{\odot}$ with effectualness of 0.90 .

Before advanced detectors begin to operate, there will be a period when LIGO and Virgo will operate with sensitivities slightly larger than but with bandwidths similar to initial detectors- the so-called Enhanced LIGO and Virgo +. Since Virgo and Virgo+ are expected to have a sensitivity bandwidth similar to Advanced LIGO, the results 
presented in this paper are qualitatively similar to those cases too. Moreover, as our results are only sensitive to the bandwidth, conclusions drawn by using the noise spectral density of Initial LIGO will also be valid for Enhanced LIGO.

All approximants (no exceptions) achieve an effectualness of 0.95 or better at 3PN and 3.5PN orders, for binaries whose total mass is less than about $\sim 12 M_{\odot}$. From the viewpoint of effectualness alone, we conclude that searches for binary black holes, in Initial, Enhanced, and Advanced LIGO, could employ any of the 3PN or 3.5PN families as long as the total mass is smaller than about $\sim 12 M_{\odot}$. The final choice of the PN family should be based on other criteria. If it is desired that the minimal match of a template bank is 0.965 or greater, then the best strategy would be to use the full EOB waveform calibrated to numerical relativity.

Another criteria to be considered is the computational cost. A typical matched filter search in LIGO data must compute thousands of template signals for every $2048 \mathrm{~s}$ data segment. This can be a heavy burden if it takes a significant amount of time to compute each template. The EOB templates are computed in the time domain by solving a set of differential equations, and the frequency domain signal is then computed via Fourier transform. For low-mass systems this cost can become significant and will of course vary depending on the implementation and hardware used.

We have estimated the cost to compute TaylorF2 and EOB templates using their implementation in the LAL code used for matched filtering searches in LIGO data. We find that for a total mass $\geq 40 M_{\odot}$, the EOB templates take a factor of 2 longer to generate than the same TaylorF2 signals. For a $(10,10) M_{\odot},(5,5) M_{\odot}$, and $(1.4,1.4) M_{\odot}$ binary, the EOB templates take about a factor of 3, 7, and 20 longer to generate, respectively. We tested the waveform generation on a high performance computer with 32 2.7 GHz CPUs and $132 \mathrm{~GB}$ of RAM. On this system, EOB templates with a total mass $\geq 40 M_{\odot}$ can be generated in about $0.1 \mathrm{~s}$, while the $(10,10) M_{\odot}$ EOB template could be generated in about $0.5 \mathrm{~s}$. Since LIGO searches employ thousands of CPUs, this is feasible. However, for lower mass signals, the time needed grows rather quickly and about $4 \mathrm{~s}$ are needed to compute the $(1.4,1.4) M_{\odot}$ EOB template. It may be possible to reduce the computational cost somewhat by optimizing the EOB waveform generation code, but the lowest mass templates would almost certainly still have a significant computational cost. Thus, the increased computational cost must be weighed against the benefit of increased effectualness for lower mass signals.

\section{FAITHFULNESS}

For completeness, we also report on the faithfulness of the different $\mathrm{PN}$ approximants with respect to one another.
The faithfulness is the overlap between normalized template and signal approximants when maximizing only over the time and phase at coalescence, $t_{C}$ and $\phi_{C}$. In Tables II and III, we list the faithfulness for each pair of PN approximants at their highest $\mathrm{PN}$ order, that is, 3.5PN order, except for the EOB model which uses a p4PN order coefficient, for both Initial and Advanced LIGO and for each of our reference binaries.

In the first row and column of the top panel of Table II, notice that every approximant has an overlap of at least 0.97 with the EOB model for both Initial and Advanced LIGO. That all approximants have good agreement for a low-mass binary without searching over mass parameters is further evidence that the 3.5PN approximants are rather close to one another during the adiabatic inspiral. Note that the T2, T3, T4, and F2 approximants all have a faithfulness

TABLE II. Faithfulness of different approximants for $(1.42,1.38) M_{\odot}$ (top panel) and $(5.2,4.8) M_{\odot}$ (bottom panel) binaries. The rows label template approximants, while the columns label signal approximants. For each pair, the top number is for Initial LIGO while the bottom number is for Advanced LIGO. All approximants are at 3.5PN order, except our EOB model which has a $\mathrm{p} 4 \mathrm{PN}$ coefficient.

\begin{tabular}{llllllll}
\hline \hline & EOB & T1 & T2 & T3 & T4 & Et & F2 \\
\hline EOB & 1 & 0.969 & 0.994 & 0.997 & 0.990 & 0.970 & 0.994 \\
& 1 & 0.971 & 0.996 & 0.998 & 0.991 & 0.974 & 0.996 \\
T1 & 0.969 & 1 & 0.982 & 0.981 & 0.987 & 0.928 & 0.982 \\
& 0.971 & 1 & 0.984 & 0.983 & 0.990 & 0.920 & 0.984 \\
T2 & 0.994 & 0.982 & 1 & 0.998 & 0.999 & 0.958 & 1.000 \\
& 0.996 & 0.984 & 1 & 0.999 & 0.999 & 0.961 & 1.000 \\
T3 & 0.997 & 0.981 & 0.998 & 1 & 0.997 & 0.959 & 0.998 \\
& 0.998 & 0.983 & 0.999 & 1 & 0.998 & 0.961 & 0.999 \\
T4 & 0.990 & 0.987 & 0.999 & 0.997 & 1 & 0.950 & 0.999 \\
& 0.991 & 0.990 & 0.999 & 0.998 & 1 & 0.949 & 0.999 \\
Et & 0.970 & 0.928 & 0.958 & 0.959 & 0.950 & 1 & 0.958 \\
& 0.974 & 0.920 & 0.961 & 0.961 & 0.949 & 1 & 0.961 \\
F2 & 0.994 & 0.982 & 1.000 & 0.998 & 0.999 & 0.958 & 1 \\
& 0.996 & 0.984 & 1.000 & 0.999 & 0.999 & 0.961 & 1 \\
\hline \hline & & & & & & & \\
\hline \hline & EOB & T1 & T2 & T3 & T4 & Et & F2 \\
\hline EOB & 1 & 0.916 & 0.974 & 0.938 & 0.981 & 0.888 & 0.970 \\
& 1 & 0.877 & 0.973 & 0.928 & 0.978 & 0.841 & 0.968 \\
T1 & 0.916 & 1 & 0.974 & 0.926 & 0.964 & 0.784 & 0.975 \\
& 0.877 & 1 & 0.955 & 0.892 & 0.947 & 0.653 & 0.957 \\
T2 & 0.974 & 0.974 & 1 & 0.949 & 0.993 & 0.861 & 0.993 \\
& 0.973 & 0.955 & 1 & 0.932 & 0.994 & 0.775 & 0.995 \\
T3 & 0.938 & 0.926 & 0.949 & 1 & 0.943 & 0.925 & 0.944 \\
& 0.928 & 0.892 & 0.932 & 1 & 0.926 & 0.876 & 0.930 \\
& 0.981 & 0.963 & 0.993 & 0.943 & 1 & 0.854 & 0.995 \\
& 0.978 & 0.947 & 0.994 & 0.926 & 1 & 0.766 & 0.996 \\
& 0.888 & 0.785 & 0.861 & 0.925 & 0.854 & 1 & 0.852 \\
& 0.841 & 0.653 & 0.775 & 0.876 & 0.767 & 1 & 0.770 \\
& 0.970 & 0.975 & 0.993 & 0.944 & 0.995 & 0.853 & 1 \\
& 0.968 & 0.957 & 0.995 & 0.930 & 0.996 & 0.770 & 1 \\
\hline \multirow{2}{*}{ T4 } & & & & & & &
\end{tabular}


$\geq 0.99$ with the EOB model, while the T1 and Et approximants have somewhat worse agreement at about 0.97 . For each pair, the faithfulness for Initial and Advanced LIGO is quite similar for these low-mass binaries.

In the bottom panel of Table II, we increase the total mass to $10 M_{\odot}$ while keeping the mass ratio nearly equal. The faithfulness drops for every pair of approximants as the merger begins to enter the sensitive band. Recall that for these masses, all pairs of approximants can achieve an effectualness of at least 0.95 by searching over the mass parameters. When we fix the masses, the T2, T4, and F2 approximants still have very good agreement with the EOB model, with faithfulness of 0.97-0.98. The EOB-T3 faithfulness has degraded somewhat to 0.93-0.94, and the Et and $\mathrm{T} 1$ approximants have rather poor agreement with the EOB model with faithfulness in the range 0.84-0.92. Note that the faithfulness is typically lower for Advanced LIGO than for Initial LIGO. We attribute this to the signals having a longer duration (and thus more time to accumulate a phase difference) in Advanced LIGO's wider sensitivity band.

TABLE III. Same as Table II but for $(10.5,9.5) M_{\odot}$ (top panel) and $(10,1.4) M_{\odot}$ (bottom panel) binaries.

\begin{tabular}{|c|c|c|c|c|c|c|c|}
\hline & EOB & T1 & $\mathrm{T} 2$ & T3 & $\mathrm{T} 4$ & Et & $\mathrm{F} 2$ \\
\hline \multirow[t]{2}{*}{ EOB } & 1 & 0.877 & 0.882 & 0.650 & 0.923 & 0.860 & 0.910 \\
\hline & 1 & 0.811 & 0.864 & 0.721 & 0.910 & 0.775 & 0.889 \\
\hline \multirow[t]{2}{*}{$\mathrm{T} 1$} & 0.877 & 1 & 0.972 & 0.712 & 0.970 & 0.817 & 0.982 \\
\hline & 0.811 & 1 & 0.955 & 0.785 & 0.943 & 0.638 & 0.966 \\
\hline \multirow[t]{2}{*}{$\mathrm{T} 2$} & 0.882 & 0.972 & 1 & 0.742 & 0.968 & 0.886 & 0.959 \\
\hline & 0.864 & 0.955 & 1 & 0.831 & 0.969 & 0.784 & 0.959 \\
\hline \multirow[t]{2}{*}{ T3 } & 0.650 & 0.712 & 0.742 & 1 & 0.707 & 0.716 & 0.709 \\
\hline & 0.721 & 0.785 & 0.831 & 1 & 0.794 & 0.782 & 0.790 \\
\hline \multirow[t]{2}{*}{$\mathrm{T} 4$} & 0.923 & 0.971 & 0.968 & 0.707 & 1 & 0.906 & 0.986 \\
\hline & 0.910 & 0.943 & 0.970 & 0.794 & 1 & 0.785 & 0.988 \\
\hline \multirow[t]{2}{*}{ Et } & 0.859 & 0.817 & 0.886 & 0.716 & 0.906 & 1 & 0.845 \\
\hline & 0.776 & 0.639 & 0.784 & 0.783 & 0.785 & 1 & 0.707 \\
\hline \multirow[t]{3}{*}{$\mathrm{F} 2$} & 0.909 & 0.982 & 0.959 & 0.708 & 0.985 & 0.846 & 1 \\
\hline & 0.889 & 0.967 & 0.959 & 0.790 & 0.988 & 0.706 & 1 \\
\hline & EOB & T1 & $\mathrm{T} 2$ & T3 & $\mathrm{T} 4$ & Et & F2 \\
\hline \multirow[t]{2}{*}{ EOB } & 1 & 0.977 & 0.973 & 0.817 & 0.859 & 0.526 & 0.990 \\
\hline & 1 & 0.959 & 0.972 & 0.801 & 0.797 & 0.413 & 0.993 \\
\hline \multirow[t]{2}{*}{$\mathrm{T} 1$} & 0.977 & 1 & 0.972 & 0.796 & 0.805 & 0.508 & 0.991 \\
\hline & 0.959 & 1 & 0.954 & 0.753 & 0.691 & 0.398 & 0.978 \\
\hline \multirow[t]{2}{*}{$\mathrm{T} 2$} & 0.973 & 0.972 & 1 & 0.835 & 0.894 & 0.543 & 0.980 \\
\hline & 0.972 & 0.954 & 1 & 0.820 & 0.834 & 0.430 & 0.976 \\
\hline \multirow[t]{2}{*}{ T3 } & 0.817 & 0.796 & 0.835 & 1 & 0.851 & 0.778 & 0.818 \\
\hline & 0.801 & 0.753 & 0.820 & 1 & 0.841 & 0.631 & 0.798 \\
\hline \multirow[t]{2}{*}{$\mathrm{T} 4$} & 0.859 & 0.805 & 0.894 & 0.851 & 1 & 0.595 & 0.852 \\
\hline & 0.797 & 0.691 & 0.834 & 0.841 & 1 & 0.456 & 0.779 \\
\hline \multirow[t]{2}{*}{ Et } & 0.526 & 0.508 & 0.543 & 0.778 & 0.595 & 1 & 0.525 \\
\hline & 0.413 & 0.398 & 0.430 & 0.631 & 0.456 & 1 & 0.411 \\
\hline \multirow[t]{2}{*}{$\mathrm{F} 2$} & 0.990 & 0.991 & 0.980 & 0.818 & 0.852 & 0.525 & 1 \\
\hline & 0.993 & 0.978 & 0.976 & 0.799 & 0.779 & 0.411 & 1 \\
\hline
\end{tabular}

In the top panel of Table III, we increase the total mass to $20 M_{\odot}$ while again keeping the mass ratio nearly equal. Once again, the faithfulness drops for all cases as the merger and ringdown become more important. The T4 and F2 approximants have the best agreement with EOB; they are the only approximants to achieve an overlap greater than 0.9 with EOB. The overlap between T3 and EOB has dropped dramatically to 0.65 and 0.72 for Initial and Advanced LIGO, respectively.

The bottom panel of Table III gives the faithfulness for each approximant pair for an asymmetric $(10,1.4) M_{\odot}$ binary. The EOB-F2 faithfulness is very good at 0.99 . The T1 and T2 approximants also have good agreement with the EOB model with faithfulness $0.96-0.98$. The T3 and T4 have poor agreement with the EOB model with faithfulness $0.80-0.86$. For this mass pair, the Et approximant has very poor agreement with all of the others; the faithfulness is $\leq 0.60$ for every approximant except $\mathrm{T} 3$.

We see a clear trend of decreasing faithfulness as the total mass of the binary increases. This is due to the late inspiral, merger, and ringdown moving into the sensitive band and becoming more important for higher mass binaries. The faithfulness is typically lower for Advanced LIGO than Initial LIGO due to its broader sensitive band. The faithfulness can vary with mass ratio. For example, for the $(10,1.4) M_{\odot}$ binary, the $\mathrm{T} 1$ and $\mathrm{T} 2$ approximants have a better faithfulness with the EOB model than the T4 approximant. However, for the nearly equal-mass binaries, the T4 approximant has the greater faithfulness with the EOB model. The TaylorF2 approximant is generally the most faithful to the EOB approximant, with one of the highest overlaps in each case. This is another argument for using TaylorF2 templates in the mass regime where EOB templates are too computationally expensive to be employed in a matched filtering search.

\section{CONCLUSIONS}

In this paper we have examined the mutual effectualness of the different families of PN approximants with a view to validate their closeness for use in the construction of search templates for compact binaries in Initial, Enhanced, and Advanced LIGO. We considered seven different approximants, each at three different PN orders, a total of 21 waveforms in all. We computed the effectualness of each of the waveforms with every other at 2PN, 3PN, and 3.5PN orders by using a template bank constructed with a minimal match of 0.99 and Initial and Advanced LIGO noise power spectral densities. Our results from a sample of four binaries show that different PN approximations are consistent with one another at 3PN and 3.5PN orders. They begin to differ only when the mass becomes so large that the plunge phase, not contained in standard PN waveforms in the adiabatic approximation, enters the detector band.

The above conclusion is best summarized by Fig. 8, where we plot the effectualness of the various PN approx- 

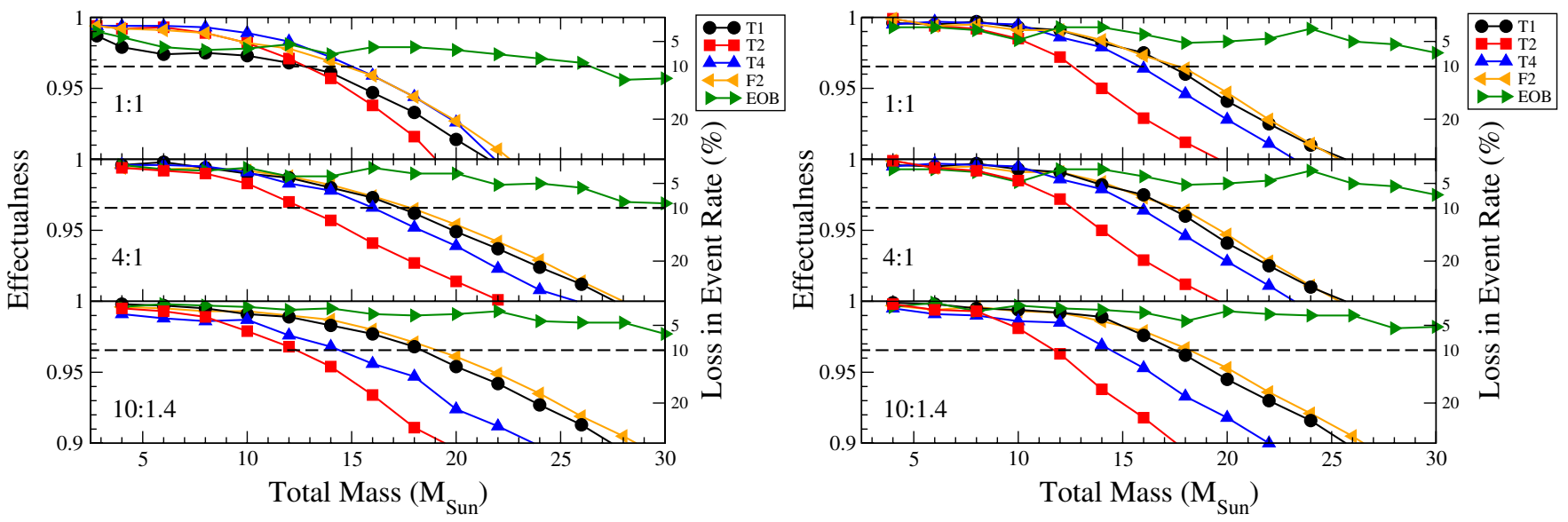

FIG. 8 (color online). Effectualness (left $y$ axis) and the corresponding loss in event rate (right $y$ axis) of 3.5PN approximants with the EOB inspiral-merger-ringdown signal calibrated to numerical relativity in Initial LIGO (left panel) and Advanced LIGO (right panel) as a function of total mass for 1:1, 4:1, and 10:1.4 mass ratios. The EOB curve is the effectualness between the uncalibrated 3.5PN EOB model containing only the inspiral and the calibrated inspiral-merger-ringdown EOB signal. Note that any of these approximants are suitable for detection templates below a total mass of about $12 M_{\odot}$ for both Initial LIGO and Advanced LIGO, provided a $10 \%$ loss of event rate is deemed acceptable.

imants (except for TaylorT3 and TaylorEt that we recommend be discarded, since we have shown that not only do they differ considerably from the others but importantly have poorer overlaps with EOB inspiral-merger-ringdown waveforms) with an EOB inspiral-merger-ringdown signal as a function of the total mass of the binary. These plots are convenient for identifying the $M_{\text {crit }}$ above which the PN approximants begin to differ with one another. We find that any of the above approximants could be used as detection templates with less than a $10 \%$ loss in event rate up to a total mass of $12 M_{\odot}$ for both Initial and Advanced LIGO. Note that this value of $M_{\text {crit }}$ is limited by the equal-mass case, as the value of $M_{\text {crit }}$ corresponding to a $10 \%$ loss in event rate is somewhat greater for mass ratios of $4: 1$ and 10:1.4. We attribute this result to asymmetric binaries accumulating more signal at low frequencies than in the equal-mass case. Thus, for a fixed total mass, the merger and ringdown are less important for asymmetric binaries than for equal-mass binaries. Therefore, we conclude that we can safely use any of the above 3.5PN families as search templates to detect binaries whose total mass is less than about $12 M_{\odot}$. However, purely from the point of view of computational burden, TaylorF2 is the least expensive and we recommend that TaylorF2 at $3.5 \mathrm{PN}$ order be deployed as a search template below a total mass of $12 M_{\odot}$. It is quite remarkable to note that up to a total mass of $30 M_{\odot}$, the uncalibrated EOB model at 3.5PN order is rather close to the calibrated EOB inspiral-mergerringdown signal. In fact, Ref. [44] found a phase difference of only $0.05 \mathrm{rad}$ after 30 gravitational-wave cycles, at roughly 3 gravitational-wave cycles before merger between the EOB at 3.5PN order and the highly accurate equal-mass numerical waveform of Caltech/Cornell Collaboration.
For systems with total mass larger than about $12 M_{\odot}$, TaylorF2 at 3.5PN might be effectual if the upper cutoff frequency is artificially extended to a higher frequency. However, this might require a tweaking of the upper frequency cutoff depending on the noise spectral density of the detector [37] and the mass ratio of the system, and either the extension to unphysical values of $\nu[36,37]$ or the inclusion of a p4PN term in the template phase [36] calibrated to the numerical simulations. We believe that a better alternative for heavier systems are the EOB templates calibrated to numerical-relativity simulations [36,38-46]. The most recent EOB models are in near perfect agreement with the most accurate numerical simulations to date, although only a small number of systems corresponding to different mass ratios have been studied so far. Nevertheless, a physical model with physically meaningful parameters is a far safer bet as search templates unless, of course, the model in question is not in agreement with the waveform predicted by numerical relativity. So far, the EOB is the best physical model we have, and this is what we recommend be used to search for binaries with masses greater than about $12 M_{\odot}$.

In this paper we adopted the preliminary, fiducial EOB model of Ref. [40], because it is the EOB model currently available in LAL and it is used for searches by Initial LIGO. For completeness, here we quantify the closeness between the EOB model used in this paper and a most recent improved version of the EOB model [46] (which is similar to the one of Ref. [45]). The latter was calibrated to longer and more accurate numerical waveforms generated by the Caltech/Cornell pseudospectral code [81]. Reference [46] found that the faithfulness of the improved EOB model to these highly accurate numerical waveforms is better than 0.999. In Table IV, we show both the faithful- 
TABLE IV. Effectualness and faithfulness of the EOB fiducial model [40] used in this paper (and currently employed by Initial LIGO) to the most recently improved EOB model [46]. We also show the bias in the parameters $M$ and $\nu$ when achieving the effectualness. For each pair, the top number is for Initial LIGO while the bottom number is for Advanced LIGO. The sign of the bias is such that in all cases the fiducial EOB templates slightly overestimate the total mass $M$ and underestimate the mass ratio $\nu$ of the improved EOB signal.

\begin{tabular}{lcccc}
\hline \hline & Effectualness & $\Delta M / M$ & $\Delta \nu / \nu$ & Faithfulness \\
\hline$(1.4,1.4) M_{\odot}$ & 0.999 & $0.98 \%$ & $-1.63 \%$ & 0.992 \\
& 0.999 & $0.98 \%$ & $-1.63 \%$ & 0.995 \\
$(1.38,1.42) M_{\odot}$ & 0.999 & $0.96 \%$ & $-1.60 \%$ & 0.992 \\
& 0.999 & $0.89 \%$ & $-1.49 \%$ & 0.995 \\
$(5,5) M_{\odot}$ & 0.997 & $1.32 \%$ & $-2.12 \%$ & 0.973 \\
$(4.8,5.2) M_{\odot}$ & 0.999 & $2.06 \%$ & $-3.47 \%$ & 0.976 \\
$(10,10) M_{\odot}$ & 0.999 & $2.42 \%$ & $-4.08 \%$ & 0.973 \\
& 0.999 & $2.11 \%$ & $-3.54 \%$ & 0.976 \\
$(9.5,10.5) M_{\odot}$ & 0.999 & $2.70 \%$ & $-4.62 \%$ & 0.974 \\
$(15,15) M_{\odot}$ & 0.999 & $2.59 \%$ & $-4.39 \%$ & 0.962 \\
& 0.998 & $1.40 \%$ & $-1.94 \%$ & 0.974 \\
$(25,25) M_{\odot}$ & 0.997 & $2.67 \%$ & $-4.54 \%$ & 0.964 \\
& 0.995 & $4.80 \%$ & $-9.98 \%$ & 0.987 \\
\hline \hline
\end{tabular}

ness and the effectualness of the EOB model [40] to the improved EOB model [46] using noise spectral densities of Initial LIGO, as well as the bias in the parameters $M$ and $\nu$ when achieving the effectualness. The search for effectualness in this test is done continuously in the parameter space, instead of using a template bank. Although there is some systematic trend in the numbers due to the difference in the EOB models, the main result is that the faithfulness and the effectualness are always better than 0.97 and 0.995 , respectively. Assuming the numerical waveforms of Ref. [46] are exact, the EOB model of Ref. [40] used in this paper is accurate for detection purposes, with a loss of event rates of $\sim 10 \%$, and may cause $\sim 10 \%$ bias in estimating the mass parameters.

In this study we considered PN waveforms in the socalled restricted PN approximation. Restricted waveforms contain only the second harmonic of the orbital frequency. Inclusion of other harmonics is necessary, especially when a binary is arbitrarily oriented with respect to a detector and the component masses are dissimilar. Recent studies $[61,62]$ have shown the tremendous advantage of including these other harmonics in the gravitational-wave templates. Therefore, it is necessary that a future effort undertakes a study similar to this, but includes all the amplitude corrections. Furthermore, Ref. [47] has shown that by supplementing the PN results by the available test particle results up to 5.5PN improves the match between the EOB models and numerical-relativity simulations. This can be expected to lead to further improvements in the results obtained here in the future.

\section{ACKNOWLEDGMENTS}

We thank Luc Blanchet for useful comments and Steve Fairhurst for discussions. We thank Michele Vallisneri for carefully reading the paper. B.R. I. thanks Cardiff University for hospitality and support during this work. A.B., E.O., and Y.P. acknowledge support from NSF Grant No. PHY-0603762.
[1] A. Abramovici et al., Science 256, 325 (1992).

[2] B. Abbott et al., Nucl. Instrum. Methods Phys. Res., Sect. A 517, 154 (2004).

[3] B. Caron et al., Classical Quantum Gravity 14, 1461 (1997).

[4] F. Acernese et al., Classical Quantum Gravity 23, S635 (2006).

[5] H. Lück et al., Classical Quantum Gravity 23, S71 (2006).

[6] K. Tsubono et al., in Gravitational Wave Detection, edited by K. Tsubono, M.-K. Fujimoto, and K. Kuroda, Frontiers Science Series Vol. 20 (Universal Academy Press, Tokyo, Japan, 1997), p. 183.

[7] J. Hough and S. Rowan, Living Rev. Relativity 3 (2000), http://www.livingreviews.org/lrr-2000-3.

[8] J.R. Smith, Classical Quantum Gravity 26, 114013 (2009).

[9] LIGO Scientific Collaboration Compact Binary Coalescence Group (private communication).

[10] L. Blanchet, Living Rev. Relativity 9, 4 (2006), http:// www.livingreviews.org/lrr-2006-4.

[11] C. Cutler, E. Poisson, G. J. Sussman, and L. S. Finn, Phys. Rev. D 47, 1511 (1993).

[12] C. Cutler and E. Flanagan, Phys. Rev. D 49, 2658 (1994).

[13] E. Poisson and C. Will, Phys. Rev. D 52, 848 (1995).

[14] T. Tanaka, H. Tagoshi, and M. Sasaki, Prog. Theor. Phys. 96, 1087 (1996).

[15] T. Damour, B. Iyer, and B. Sathyaprakash, Phys. Rev. D 57, 885 (1998).

[16] S. Droz, D. J. Knapp, E. Poisson, and B. J. Owen, Phys. Rev. D 59, 124016 (1999).

[17] T. Damour, B. R. Iyer, and B. S. Sathyaprakash, Phys. Rev. D 62, 084036 (2000).

[18] T. Damour, B. Iyer, and B. Sathyaprakash, Phys. Rev. D 63, 044023 (2001); 72, 029902(E) (2005).

[19] T. Damour, B. R. Iyer, and B. S. Sathyaprakash, Phys. Rev. D 66, 027502 (2002).

[20] T. Damour, B. R. Iyer, P. Jaranowski, and B.S. Sathyaprakash, Phys. Rev. D 67, 064028 (2003). 
[21] P. Canitrot, Phys. Rev. D 63, 082005 (2001).

[22] A. Buonanno, Y. Chen, and M. Vallisneri, Phys. Rev. D 67, 024016 (2003).

[23] A. Buonanno, Y. Chen, and M. Vallisneri, Phys. Rev. D 67, 104025 (2003).

[24] K. G. Arun, B. R. Iyer, B.S. Sathyaprakash, and P. A. Sundararajan, Phys. Rev. D 71, 084008 (2005).

[25] P. Ajith, B. R. Iyer, C. A. K. Robinson, and B.S. Sathyaprakash, Phys. Rev. D 71, 044029 (2005).

[26] L. Lindblom, B. J. Owen, and D. A. Brown, Phys. Rev. D 78, 124020 (2008).

[27] A. Buonanno and T. Damour, Phys. Rev. D 59, 084006 (1999).

[28] A. Buonanno and T. Damour, Phys. Rev. D 62, 064015 (2000).

[29] T. Damour, P. Jaranowski, and G. Schäfer, Phys. Rev. D 62, 084011 (2000).

[30] F. Pretorius, Phys. Rev. Lett. 95, 121101 (2005).

[31] M. Campanelli, C. Lousto, P. Marronetti, and Y. Zlochower, Phys. Rev. Lett. 96, 111101 (2006).

[32] J. Baker, J. Centrella, D. Choi, M. Koppitz, and J. van Meter, Phys. Rev. Lett. 96, 111102 (2006).

[33] M. Boyle et al., Phys. Rev. D 76, 124038 (2007).

[34] F. Pretorius, in Physics of Relativistic Objects in Compact Binaries: From Birth to Coalescence, edited by M. Colpi, P. Casella, V. Gorini, U. Moschella, and A. Possenti, Astrophysics and Space Science Library Vol. 359 (Springer, Berlin, New York, 2009).

[35] P. Ajith et al., Phys. Rev. D 77, 104017 (2008).

[36] Y. Pan et al., Phys. Rev. D 77, 024014 (2008).

[37] M. Boyle, D. A. Brown, and L. Pekowsky, Classical Quantum Gravity 26, 114006 (2009).

[38] A. Buonanno, G. B. Cook, and F. Pretorius, Phys. Rev. D 75, 124018 (2007).

[39] T. Damour and A. Nagar, Phys. Rev. D 76, 064028 (2007).

[40] A. Buonanno et al., Phys. Rev. D 76, 104049 (2007).

[41] T. Damour and A. Nagar, Phys. Rev. D 77, 024043 (2008).

[42] T. Damour, A. Nagar, E. N. Dorband, D. Pollney, and L. Rezzolla, Phys. Rev. D 77, 084017 (2008).

[43] T. Damour, A. Nagar, M. Hannam, S. Husa, and B. Brügmann, Phys. Rev. D 78, 044039 (2008).

[44] M. Boyle, A. Buonanno, L. E. Kidder, A. H. Mroué, Y. Pan, H. P. Pfeiffer, and M. A. Scheel, Phys. Rev. D 78, 104020 (2008).

[45] T. Damour and A. Nagar, Phys. Rev. D 79, 081503 (2009).

[46] A. Buonanno et al., Phys. Rev. D 79, 124028 (2009).

[47] T. Damour, B. R. Iyer, and A. Nagar, Phys. Rev. D 79, 064004 (2009).

[48] S. Bose, A. Gopakumar, and M. Tessmer, arXiv:0807.2400.

[49] A. Gopakumar, arXiv:0712.3236.

[50] A. Gopakumar, M. Hannam, S. Husa, and B. Brügmann, Phys. Rev. D 78, 064026 (2008).

[51] M. Tessmer and A. Gopakumar, arXiv:0812.0549.

[52] K. Martel and E. Poisson, Phys. Rev. D 60, 124008 (1999).
[53] T. Damour, A. Gopakumar, and B. R. Iyer, Phys. Rev. D 70, 064028 (2004).

[54] C. Konigsdorffer and A. Gopakumar, Phys. Rev. D 73, 124012 (2006).

[55] M. Tessmer and A. Gopakumar, Phys. Rev. D 78, 084029 (2008).

[56] K. G. Arun, L. Blanchet, B. R. Iyer, and M. S. S. Qusailah, Phys. Rev. D 77, 064034 (2008).

[57] K. G. Arun, L. Blanchet, B. R. Iyer, and M. S. S. Qusailah, Phys. Rev. D 77, 064035 (2008).

[58] N. Yunes, K. G. Arun, E. Berti, and C. M. Will, Phys. Rev. D 80, 084001 (2009).

[59] P. Peters and J. Mathews, Phys. Rev. 131, 435 (1963).

[60] P. Peters, Phys. Rev. 136, B1224 (1964).

[61] C. Van Den Broeck and A. Sengupta, Classical Quantum Gravity 24, 1089 (2007).

[62] C. Van Den Broeck and A. Sengupta, Classical Quantum Gravity 24, 155 (2007).

[63] T. Damour, P. Jaranowski, and G. Schäfer, Phys. Lett. B 513, 147 (2001).

[64] L. Blanchet, T. Damour, and G. Esposito-Farese, Phys. Rev. D 69, 124007 (2004).

[65] L. Blanchet, T. Damour, G. Esposito-Farese, and B. Iyer, Phys. Rev. Lett. 93, 091101 (2004).

[66] L. Blanchet, T. Damour, G. Esposito-Farese, and B. Iyer, Phys. Rev. D 71, 124004 (2005).

[67] T. Damour, P. Jaranowski, and G. Schäfer, Phys. Rev. D 63, 044021 (2001).

[68] V.C. de Andrade, L. Blanchet, and G. Faye, Classical Quantum Gravity 18, 753 (2001).

[69] L. Blanchet and B. R. Iyer, Classical Quantum Gravity 20, 755 (2003).

[70] Y. Itoh and T. Futamase, Phys. Rev. D 68, 121501 (2003).

[71] L. Blanchet, B. R. Iyer, and B. Joguet, Phys. Rev. D 65, 064005 (2002).

[72] L. Blanchet, G. Faye, B. R. Iyer, and B. Joguet, Phys. Rev. D 65, 061501 (2002).

[73] L. Blanchet and B. R. Iyer, Phys. Rev. D 71, 024004 (2005).

[74] L. Blanchet, T. Damour, B. Iyer, C. Will, and A. Wiseman, Phys. Rev. Lett. 74, 3515 (1995).

[75] L. Blanchet, T. Damour, and B. R. Iyer, Phys. Rev. D 51, 5360 (1995).

[76] C. M. Will and A. G. Wiseman, Phys. Rev. D 54, 4813 (1996).

[77] J. G. Baker, J.R. van Meter, S.T. McWilliams, J. Centrella, and B.J. Kelly, Phys. Rev. Lett. 99, 181101 (2007).

[78] T. Damour and A. Gopakumar, Phys. Rev. D 73, 124006 (2006).

[79] E. Berti, V. Cardoso, and C. Will, Phys. Rev. D 73, 064030 (2006).

[80] T. Cokelaer, Phys. Rev. D 76, 102004 (2007).

[81] M. A. Scheel et al., Phys. Rev. D 79, 024003 (2009). 